\title{
ERROR ANALYSIS FOR A MONOLITHIC DISCRETIZATION OF COUPLED DARCY AND STOKES PROBLEMS
}

By

Vivette Girault, Guido Kanschat, Béatrice Rivière

IMA Preprint Series \#2390

(APRIL 2012)

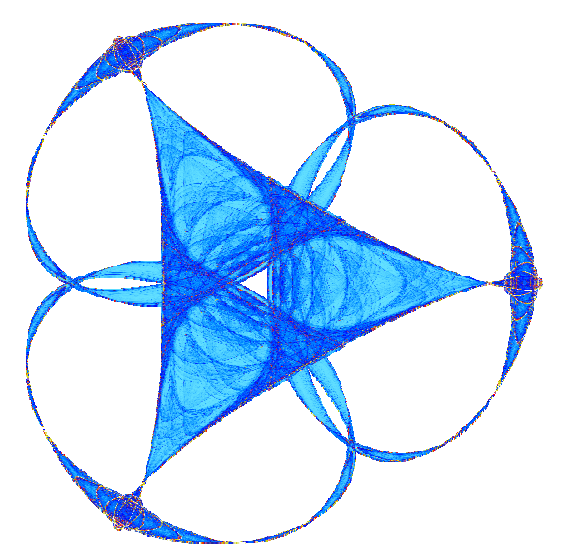

INSTITUTE FOR MATHEMATICS AND ITS APPLICATIONS

UNIVERSITY OF MINNESOTA

400 Lind Hall

207 Church Street S.E.

Minneapolis, Minnesota 55455-0436

Phone: 612-624-6066 Fax: 612-626-7370

URL: http://www.ima.umn.edu 


\section{ERROR ANALYSIS FOR A MONOLITHIC DISCRETIZATION OF COUPLED DARCY AND STOKES PROBLEMS}

By

Vivette Girault, Guido Kanschat, Béatrice Rivière

IMA Preprint Series \#2390

(APRIL 2012)

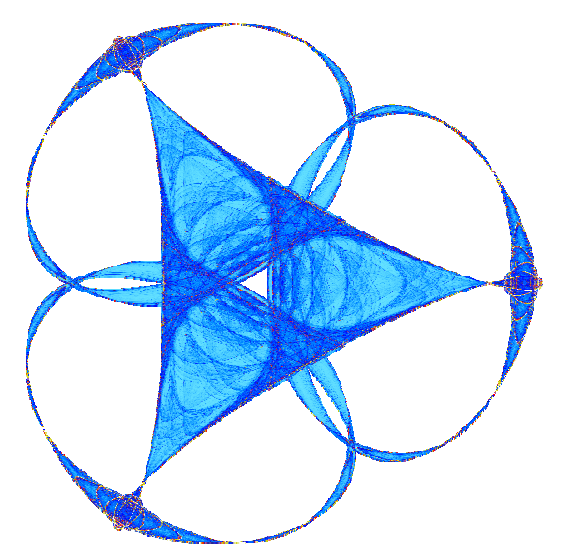

INSTITUTE FOR MATHEMATICS AND ITS APPLICATIONS

UNIVERSITY OF MINNESOTA

400 Lind Hall

207 Church Street S.E.

Minneapolis, Minnesota 55455-0436

Phone: 612-624-6066 Fax: 612-626-7370

URL: http://www.ima.umn.edu 


\title{
ERROR ANALYSIS FOR A MONOLITHIC DISCRETIZATION OF COUPLED DARCY AND STOKES PROBLEMS
}

\author{
VIVETTE GIRAULT, GUIDO KANSCHAT, AND BÉATRICE RIVIÈRE
}

\begin{abstract}
The coupled Stokes and Darcy equations are approximated by a strongly conservative finite element method. The discrete spaces are the divergence-conforming velocity space with matching pressure space such as the Raviart-Thomas spaces. This work proves optimal error estimate of the velocity in the $L^{2}$ norm in the domain and on the interface under weak regularity assumptions.
\end{abstract}

\section{INTRODUCTION}

Recently, a monolithic strongly conservative finite element method that combines the discontinuous Galerkin method for the Stokes equations with the mixed finite element method for the Darcy equations was introduced by Kanschat and Rivière [28]. Mass conservation is achieved in the $\mathbf{H}^{\text {div }}$ sense, meaning that the divergence of the velocity is pointwise equal to zero inside the mesh elements. Energy error estimates were derived in [28] but these are not optimal in the Darcy region. In fact, standard duality arguments cannot be applied. In this article, we complete the error analysis of our scheme by deriving optimal $L^{2}$ error estimates and thus providing mathematical proof of the convergence rates observed in [28]. All estimates are done with weak regularity assumptions, such that they apply to cases where the interface is not smooth. The argument is based on a sharp estimate for the trace of the solution on the interface. As a result of our analysis, we find that the orders of approximation of the velocity in the Darcy and Stokes subdomains are balanced.

Modeling of Darcy-Stokes coupling traces back to the seminal work of Beavers and Joseph [5] and Saffman [36]. The Beavers-Joseph-Saffman model can be derived by homogenization (see Jäger \& Mikelic [25] and references therein) and its well-posedness has been established by Layton et al. [29]. In the latter, a finite element method based on continuous and $\mathbf{H}^{\text {div }}$ elements in the Stokes and Darcy subdomains, respectively, is introduced. The coupling is achieved by a mortar. Alternatively, a primal formulation has been applied in the Darcy subregion (see Discacciati \& Quarteroni [15] and references therein); this formulation also allows for interface conditions involving the tangential Darcy velocity, but mass conservation is not as natural anymore. Riviere and Yotov [35] and Gatica et al [18] proposed a primal formulation in the Stokes region coupled with a dual formulation in the Darcy region, which means that different elements are used in both regions. In [17], Gatica et al. analyzed a fully mixed formulation that solves the pseudo-stress and velocity in the Stokes region and requires Lagrange multipliers. Finally, the work of Arbogast and

2010 Mathematics Subject Classification. Primary 65N30, 65N12; Secondary 76S05, 76D07.

Key words and phrases. Darcy-Stokes coupling, Beavers-Joseph-Saffman condition, mixed finite elements.

Supported in part by the National Science Foundation through grant no. DMS-0810387 and by the King Abdullah University of Science and Technology (KAUST) through award no. KUS-C1-016-04. Part of this research was prepared when the author visited the Institute for Mathematics and its Applications in Minneapolis.

Supported in part by the National Science Foundation through grant no. DMS-0810422. 
Brunson [2] uses a finite element with continuity requirements changing between $\mathbf{H}^{1}$ and $\mathbf{H}^{\text {div }}$ as needed. Our discretization is distinguished from these by

(a) employing a conservative formulation in the whole domain

(b) using the same $\mathbf{H}^{\text {div }}$ conforming element pair in the Stokes and the Darcy subdomain, and

(c) a discontinuous Galerkin method to address the resulting inconsistency in the Stokes subdomain.

Thus, it addresses the issue of mass loss reported for instance by Hanspal et al. [24] and it does not require a mortar between the subdomains. Due to the fact that the same element is used over the whole domain, it lends itself to particularly simple implementations.

The outline of the paper is as follows. Section 2 defines the model problem including the interface conditions. Across the interface, we assume continuity of normal component of velocity, balance of forces and the Beavers-Joseph-Saffman law. The numerical scheme is then defined. Instead of specifying a particular discontinuous Galerkin form for the Stokes subdomain, we make generic assumptions and show they are satisfied by the interior penalty discontinuous Galerkin methods and the local discontinuous Galerkin method. Section 3 contains the error analysis. It begins with our main result, Theorem 5, followed by the auxiliary results needed for its proof.

\section{Model Problem And Discretization}

Let $\Omega$ be a bounded Lipschitz domain in $\mathbb{R}^{d}, d=2,3$, split into two Lipschitz subregions $\Omega_{S}$ and $\Omega_{D}$ of free and porous media flow, respectively. By $\Gamma_{S}, \Gamma_{D}$, and $\Gamma_{S D}$ we denote the boundaries of the subdomains according to the definition

$$
\Gamma_{S D}=\partial \Omega_{S} \cap \partial \Omega_{D} \quad \Gamma_{S}=\partial \Omega \cap \partial \Omega_{S}, \quad \Gamma_{D}=\partial \Omega \cap \partial \Omega_{D} .
$$

When $d=3$, we assume that the boundaries of $\Gamma_{S}, \Gamma_{D}$, and $\Gamma_{S D}$ are all Lipschitz. To simplify the discussion, in the case when $\Omega_{S}$ is not connected, we assume that each of its connected components is adjacent to a portion of $\Gamma_{S}$ with positive measure. The results of this work remain true in the general situation, but some proofs are more involved. The geometry of the boundary components also determines the regularity of solutions, in particular for a nondifferentiable interface. Since we are not aware of a thorough study of the expected regularity, we derive estimates under weak assumptions, leaving the regularity of the solution as a parameter.

The coupled Darcy/Stokes problem in conservative form reads

$$
\begin{aligned}
-\nabla \cdot(2 \nu \varepsilon(\mathbf{u}))+\nabla p & =\mathbf{f}, & & \text { in } \Omega_{S}, \\
K^{-1} \mathbf{u}+\nabla p & =\mathbf{f}, & & \text { in } \Omega_{D}, \\
\nabla \cdot \mathbf{u} & =g, & & \text { in } \Omega .
\end{aligned}
$$

The deformation tensor is $\varepsilon(\mathbf{u})=\frac{1}{2}\left(\nabla \mathbf{u}+(\nabla \mathbf{u})^{T}\right)$. The coefficient $\nu>0$ is the fluid kinematic viscosity. The variable $K$ is the ratio of the intrinsic permeability to the fluid viscosity. It is a symmetric positive definite tensor with eigenvalues $\left\{\lambda_{i}\right\}_{i=1, \ldots, d}$, uniformly bounded above and away from zero. The intrinsic permeability is allowed to vary continuously over $\Omega_{D}$. The data $\mathbf{f}$ and $g$ are chosen respectively in the space of vector-valued functions $\mathbf{L}^{2}(\Omega)$ and the scalar-valued space $L^{2}(\Omega)$, with the restriction (4) below. Here, we consider the most general — not necessarily physically meaningful — case of inhomogeneous right-hand sides in both equations and both subdomains. Studying the problem in this generality will allow us for instance to apply our results to dual problems as they occur in a posteriori error estimation. Whenever we want to distinguish between the solution of 
the Stokes and the Darcy subproblem, we refer to $\mathbf{u}_{S}=\left.\mathbf{u}\right|_{\Omega_{S}}$ and $\mathbf{u}_{D}=\left.\mathbf{u}\right|_{\Omega_{D}}$ and analogue for the pressures $p_{S}$ and $p_{D}$. On the interface $\Gamma_{S D}$, this notation refers to the traces taken from the respective subdomains.

To complete this problem, we need interface and boundary conditions. For any bounded domain $\mathcal{O}$, let $\mathbf{n}$ denote the unit normal vector to $\partial \mathcal{O}$, exterior to $\mathcal{O}$; in the case of the interface $\Gamma_{S D}, \mathbf{n}$ is the unit normal vector to $\Gamma_{S D}$ directed from $\Omega_{S}$ to $\Omega_{D}$, and $\boldsymbol{\tau}^{j}, 1 \leq$ $j \leq d-1$, is an orthonormal set of tangent vectors on the tangent plane to $\Gamma_{S D}$. On the interface, we impose the Beavers-Joseph-Saffman conditions (see Beavers and Joseph [5] and Saffman [36]), and on the boundary, we assume no-slip and Neumann for simplicity:

$$
\begin{aligned}
\mathbf{u}_{S} \cdot \mathbf{n} & =\mathbf{u}_{D} \cdot \mathbf{n}, & & \text { on } \Gamma_{S D}, \\
p_{S}-2 \nu \varepsilon\left(\mathbf{u}_{S}\right) \mathbf{n} \cdot \mathbf{n} & =p_{D}, & & \text { on } \Gamma_{S D}, \\
\gamma^{2} K^{-\frac{1}{2}} \mathbf{u}_{S} \cdot \boldsymbol{\tau}^{j}+2 \nu \varepsilon\left(\mathbf{u}_{S}\right) \mathbf{n} \cdot \boldsymbol{\tau}^{j} & =0, & & \text { on } \Gamma_{S D}, j \in[1, d-1], \\
\mathbf{u}_{S} & =0, & & \text { on } \Gamma_{S}, \\
\mathbf{u}_{D} \cdot \mathbf{n} & =0, & & \text { on } \Gamma_{D} .
\end{aligned}
$$

Here, the tensor $K^{-\frac{1}{2}}$ is obtained from $K$ by replacing the eigenvalues $\lambda_{i}$ by $\lambda_{i}^{-\frac{1}{2}}$. The function $\gamma$ is a positive, smooth function on the interface bounded uniformly above and away from zero.

We use the standard notation $H^{s}(\mathcal{O})$ for the Sobolev space of order $s$ on a bounded domain $\mathcal{O}$, see Adams \& Fournier, Lions \& Magenes, or Nečas [1,30,31], as well as $\mathbf{H}^{s}(\mathcal{O})$ for spaces of vector-valued functions. When $k$ is an integer, the norm and seminorm of $H^{k}(\mathcal{O})$ are defined as usual by

$$
\|v\|_{H^{k}(\mathcal{O})}=\left(\sum_{j=0}^{k} \sum_{|\alpha|=j}\left\|\partial^{\alpha} v\right\|_{L^{2}(\mathcal{O})}^{2}\right)^{\frac{1}{2}}, \quad|v|_{H^{k}(\mathcal{O})}=\left(\sum_{|\alpha|=k}\left\|\partial^{\alpha} v\right\|_{L^{2}(\mathcal{O})}^{2}\right)^{\frac{1}{2}},
$$

respectively. We refer to [1,30,31] for the extension of these definitions when $s$ is not an integer. In order to obtain weak solutions to the set of equations (2)-(3), we introduce the spaces for the velocity part of the solution

$$
\begin{aligned}
& \mathbf{H}^{\mathrm{div}}(\Omega)=\left\{\mathbf{v} \in \mathbf{L}^{2}(\Omega) \mid \nabla \cdot \mathbf{v} \in L^{2}(\Omega)\right\}, \\
& \mathbf{H}_{0}^{\mathrm{div}}(\Omega)=\left\{\mathbf{v} \in \mathbf{H}^{\mathrm{div}}(\Omega) \mid \mathbf{v} \cdot \mathbf{n}=0 \text { on } \partial \Omega\right\},
\end{aligned}
$$

see for instance Girault \& Raviart [19]. Since the domain of the Stokes operator in (2a) is $\mathbf{H}^{1}\left(\Omega_{S}\right)$ for the velocity, we have to require additional differentiability. To begin with, it is easy to check on the one hand that equations (2)-(3) are unchanged if an arbitrary constant is added to the pressure $p$. We choose this undetermined constant by prescribing that

$$
\int_{\Omega} p=0
$$

On the other hand, the boundary conditions (3d) and (3e) imply that

$$
\int_{\Omega} \nabla \cdot \mathbf{u}=0
$$

Therefore $g$ must satisfy the compatibility condition

$$
\int_{\Omega} g=0 .
$$


Thus, the function spaces for our weak formulation are

$$
\begin{aligned}
& \mathbf{V}=\left\{\mathbf{v} \in \mathbf{H}_{0}^{\mathrm{div}}(\Omega)|\mathbf{v}|_{\Omega_{S}} \in \mathbf{H}^{1}\left(\Omega_{s}\right) \text { and }\left.\mathbf{v}\right|_{\Gamma_{S}}=\mathbf{0}\right\}, \\
& Q=\left\{q \in L^{2}(\Omega) \mid \int_{\Omega} q=0\right\}=L_{0}^{2}(\Omega) .
\end{aligned}
$$

The restriction of $\mathbf{V}$ to $\Omega_{S}$ is denoted by $\mathbf{V}_{S}$. The space $\mathbf{V}_{D}$ is defined in section 3.4. We also introduce the subspace of divergence-free vectors $\stackrel{\circ}{\mathbf{V}}$ of $\mathbf{V}$ :

$$
\stackrel{\circ}{\mathbf{V}}=\{\mathbf{v} \in \mathbf{V} \mid \nabla \cdot \mathbf{v}=0\} .
$$

The measure of a bounded set $\mathcal{O}$ is denoted by $|\mathcal{O}|$. We use the scalar product notation and norm on $\Omega$, boundaries, faces, and subsets of those, namely

$$
\begin{aligned}
(\phi, \psi)_{\Omega} & =\int_{\Omega} \phi \odot \psi d \mathbf{x}, & \langle\phi, \psi\rangle_{\Gamma} & =\int_{\Gamma} \phi \odot \psi d s, \\
\|\phi\|_{L^{2}(\Omega)} & =\left(\int_{\Omega}|\phi|^{2} d \mathbf{x}\right)^{\frac{1}{2}}, & \|\phi\|_{L^{2}(\Gamma)} & =\left(\int_{\Gamma}|\phi|^{2} d s\right)^{\frac{1}{2}} .
\end{aligned}
$$

The pointwise multiplication operator $\phi \odot \psi$ refers to the product $\phi \psi$, the scalar product $\phi \cdot \psi$ and the double contraction $\phi: \psi$ for scalar, vector and tensor arguments, respectively. The modulus $|\phi|=\sqrt{\phi \odot \phi}$ is defined accordingly. The space $Q$ is equipped with the $L^{2}$ norm in $\Omega$, the space $\mathbf{V}$ has the norm

$$
\forall \mathbf{v} \in \mathbf{V},\|\mathbf{v}\|_{\mathbf{v}}=\left(\left\|K^{-\frac{1}{2}} \mathbf{v}\right\|_{\mathbf{L}^{2}\left(\Omega_{D}\right)}^{2}+\|\nabla \cdot \mathbf{v}\|_{L^{2}(\Omega)}^{2}+\|\nabla \mathbf{v}\|_{\mathbf{L}^{2}\left(\Omega_{S}\right)}^{2}\right)^{\frac{1}{2}},
$$

and is a Hilbert space for this norm, owing to Poincaré's inequality.

2.1. Weak formulation. For $\mathbf{u}, \mathbf{v} \in \mathbf{V}$ and $q \in Q$, we introduce the bilinear forms

$$
\begin{aligned}
a_{D}(\mathbf{u}, \mathbf{v}) & =\left(K^{-1} \mathbf{u}, \mathbf{v}\right)_{\Omega_{D}}, \\
a_{S}(\mathbf{u}, \mathbf{v}) & =2 \nu(\varepsilon(\mathbf{u}), \varepsilon(\mathbf{v}))_{\Omega_{S}}, \\
a_{I}(\mathbf{u}, \mathbf{v}) & =\sum_{j=1}^{d-1}\left\langle\gamma^{2} K^{-\frac{1}{2}} \mathbf{u}_{S} \cdot \boldsymbol{\tau}^{j}, \mathbf{v}_{S} \cdot \boldsymbol{\tau}^{j}\right\rangle_{\Gamma_{S D}}, \\
a(\mathbf{u}, \mathbf{v}) & =a_{D}(\mathbf{u}, \mathbf{v})+a_{I}(\mathbf{u}, \mathbf{v})+a_{S}(\mathbf{u}, \mathbf{v}), \\
b(\mathbf{v}, q) & =-(\nabla \cdot \mathbf{v}, q)_{\Omega} .
\end{aligned}
$$

The weak formulation of problem (2)-(3) reads: Find $(\mathbf{u}, p) \in \mathbf{V} \times Q$ such that

$$
\begin{aligned}
a(\mathbf{u}, \mathbf{v})+b(\mathbf{v}, p) & =(\mathbf{f}, \mathbf{v})_{\Omega}, & & \forall \mathbf{v} \in \mathbf{V}, \\
b(\mathbf{u}, q) & =-(g, q)_{\Omega}, & & \forall q \in Q .
\end{aligned}
$$

Note that since both $\nabla \cdot \mathbf{u}$ and $g$ belong to $L_{0}^{2}(\Omega)$, we can relax the zero mean value constraint on the test functions $q$ and this last equation is valid for all $q$ in $L^{2}(\Omega)$. It can be shown that the weak formulation (7) is equivalent to the boundary value problem (2)-(3); see Girault \& Rivière [20] for an interpretation of the boundary conditions on the interface. The bilinear form $b(\cdot, \cdot)$ satisfies an inf-sup condition that easily follows from the standard inf-sup condition between $\mathbf{H}_{0}^{1}(\Omega)$ and $L_{0}^{2}(\Omega)$ (see for instance [19]): There exists a constant $\kappa>0$ such that

$$
\forall q \in L_{0}^{2}(\Omega), \sup _{\mathbf{v} \in \mathbf{H}_{0}^{1}(\Omega)} \frac{b(\mathbf{v}, q)}{|\mathbf{v}|_{\mathbf{H}^{1}(\Omega)}} \geq \kappa\|q\|_{L^{2}(\Omega)} .
$$


As $\mathbf{H}_{0}^{1}(\Omega) \subset \mathbf{V}$ with continuous imbedding, (8) immediately implies that there exists a constant $\beta>0$ such that

$$
\forall q \in L_{0}^{2}(\Omega), \sup _{\mathbf{v} \in \mathbf{V}} \frac{b(\mathbf{v}, q)}{\|\mathbf{v}\|_{\mathbf{v}}} \geq \beta\|q\|_{L^{2}(\Omega)} .
$$

The first inf-sup condition (8) allows in particular to lift the nonzero divergence constraint:

Proposition 1. Given $g$ in $L_{0}^{2}(\Omega)$, there is a function $\mathbf{u}_{g}$ in $\mathbf{H}_{0}^{1}(\Omega)$ such that

$$
\nabla \cdot \mathbf{u}_{g}=g \quad\left|\mathbf{u}_{g}\right|_{H^{1}(\Omega)} \leq \frac{1}{\kappa}\|g\|_{L^{2}(\Omega)} .
$$

This leads to the well-posedness of (7). Indeed the auxiliary function $\stackrel{\circ}{\mathbf{u}}=\mathbf{u}-\mathbf{u}_{g}$ solves the following problem that is obviously equivalent to (7):

$$
\begin{aligned}
a\left({ }_{\mathbf{u}}^{\circ}, \mathbf{v}\right)+b(\mathbf{v}, p) & =(\mathbf{f}, \mathbf{v})_{\Omega}-a\left(\mathbf{u}_{g}, \mathbf{v}\right), & & \forall \mathbf{v} \in \mathbf{V}, \\
b(\stackrel{\circ}{\mathbf{u}}, q) & = & 0, & \forall q \in Q .
\end{aligned}
$$

Moreover, considering that Korn's inequality holds for functions in $\mathbf{H}^{1}\left(\Omega_{S}\right)$ that vanish on $\Gamma_{S}$, the bilinear form $a(\cdot, \cdot)$ is elliptic and continuous on $\mathbf{V} \times \mathbf{V}$. Therefore a straightforward application of the Babuška-Brezzi theory (see for instance [19]) shows that problem (11) is well-posed, i.e. it has a unique solution that depends continuously on the data.

In the analysis that follows, we make use of an adjoint problem defined as follows: for $\boldsymbol{\psi}$ given in $\mathbf{L}^{2}\left(\Omega_{S}\right)$, find $\left(\mathbf{u}^{*}, p^{*}\right)$ in $\mathbf{V} \times Q$, solution of

$$
\begin{aligned}
a\left(\mathbf{v}, \mathbf{u}^{*}\right)+b\left(\mathbf{v}, p^{*}\right) & =(\mathbf{v}, \psi)_{\Omega_{S}}, & & \forall \mathbf{v} \in \mathbf{V}, \\
b\left(\mathbf{u}^{*}, q\right) & =0, & & \forall q \in Q .
\end{aligned}
$$

This problem has a unique weak solution, and since the form $a(\cdot, \cdot)$ is symmetric, this solution $\left(\mathbf{u}^{*}, p^{*}\right)$ satisfies the interior equations (2) with $\mathbf{f}=\boldsymbol{\psi}$ in $\Omega_{S}, \mathbf{f}=\mathbf{0}$ in $\Omega_{D}$, and $g=0$, namely:

$$
\begin{aligned}
-\nabla \cdot\left(2 \nu \varepsilon\left(\mathbf{u}^{*}\right)\right)+\nabla p^{*} & =\boldsymbol{\psi}, & & \text { in } \Omega_{S}, \\
K^{-1} \mathbf{u}^{*}+\nabla p^{*} & =\mathbf{0}, & & \text { in } \Omega_{D}, \\
\nabla \cdot \mathbf{u}^{*} & =0, & & \text { in } \Omega,
\end{aligned}
$$

the interface conditions (3a)-(3c) and the boundary conditions (3d)-(3e). We make the following assumption on its regularity.

Assumption 1. The boundaries $\Gamma_{S}, \Gamma_{D}$ and $\Gamma_{S D}$ are such that, for some real number $\alpha$, $\frac{1}{2}<\alpha \leq 1,\left(\mathbf{u}^{*}, p^{*}\right)$ can be estimated by

$$
\begin{aligned}
\left\|\mathbf{u}_{S}^{*}\right\|_{H^{1+\alpha}\left(\Omega_{S}\right)} & \leq C\|\boldsymbol{\psi}\|_{\mathbf{L}^{2}\left(\Omega_{S}\right)}, & \left\|\mathbf{u}_{D}^{*}\right\|_{H^{\alpha}\left(\Omega_{D}\right)} & \leq C\|\boldsymbol{\psi}\|_{\mathbf{L}^{2}\left(\Omega_{S}\right)}, \\
\left\|p_{S}^{*}\right\|_{H^{\alpha}\left(\Omega_{S}\right)} & \leq C\|\boldsymbol{\psi}\|_{\mathbf{L}^{2}\left(\Omega_{S}\right)}, & \left\|p_{D}^{*}\right\|_{H^{1+\alpha}\left(\Omega_{D}\right)} & \leq C\|\boldsymbol{\psi}\|_{\mathbf{L}^{2}\left(\Omega_{S}\right)}
\end{aligned}
$$

Note that the assumption on $p_{D}^{*}$ follows immediately from the assumption on $\mathbf{u}_{D}^{*}$.

2.2. Discretization. From now on, we assume that $\Omega_{S}$ and $\Omega_{D}$ are polygons or Lipschitz polyhedra, according to the dimension. Let $\mathbb{T}_{h}$ be a regular family (in the sense of Ciarlet [11]) of conforming subdivisions of $\bar{\Omega}$ into simplices, parallelograms or parallepipeds, such that the interface $\Gamma_{S D}$ is the union of element faces. The case of arbitrary quadrilaterals or hexahedra is more complex because of the anisotropic behavior of the Piola transform (see Arnold, Boffi, and Falk [3]); all caveats in this reference apply to our method as well. For any element $T \in \mathbb{T}_{h}$, we denote by $h_{T}$ its diameter, by $\rho_{T}$, the maximum diameter of 
spheres inscribed in $T$, and by $h$ the maximum diameter over all mesh elements. The regularity assumption on the family $\mathbb{T}_{h}$ states that there exists a constant $\theta>0$, independent of $h$ such that

$$
\forall T \in \mathbb{T}_{h}, \frac{h_{T}}{\rho_{T}} \leq \theta .
$$

Additionally, we introduce the notation $\mathbb{T}_{h}^{S}$ for all cells of $\mathbb{T}_{h}$ which lie in $\overline{\Omega_{S}}$. Denote by $\Gamma_{i}^{S}$ the set of faces that are interior to $\Omega_{S}$ and by $\Gamma_{b}^{S}$ the set of those that lie on the boundary $\Gamma_{S}$, furthermore let $\Gamma_{h}^{S}=\Gamma_{b}^{S} \cup \Gamma_{i}^{S}$.

We use the same convention as above to denote the scalar products and norms of vectors or tensors, but since we have in mind a DG method for the Stokes operator, we introduce suitable norms on the cells and faces of $\mathbb{T}_{h}$ and $\Gamma_{h}^{S}$; thus we define analogously to (5)

$$
\begin{aligned}
(\phi, \psi)_{\mathbb{T}_{h}} & =\sum_{T \in \mathbb{T}_{h}} \int_{T} \phi \odot \psi d \mathbf{x}, & \langle\phi, \psi\rangle_{\Gamma_{*}^{S}} & =\sum_{F \in \Gamma_{*}^{S}} \int_{F} \phi \odot \psi d s, \\
\|\phi\|_{\mathbb{T}_{h}} & =\left(\sum_{T \in \mathbb{T}_{h}} \int_{T}|\phi|^{2} d \mathbf{x}\right)^{\frac{1}{2}}, & \|\phi\|_{\Gamma_{*}^{S}} & =\left(\sum_{F \in \Gamma_{*}^{S}} \int_{F}|\phi|^{2} d s\right)^{\frac{1}{2}},
\end{aligned}
$$

where the index $\star$ stands for $b, i$, or $h$.

2.2.1. Discretization spaces and projections. For each integer $k \geq 0$, let $\mathbb{P}_{k}$ be the space of polynomials in two or three variables of total degree less than or equal to $k$, and $\mathbb{Q}_{k}$ the space of polynomials of degree less than or equal to $k$ in each variable. For the discrete spaces, we use pairs of a divergence-conforming velocity space $\mathbf{V}_{h} \subset \mathbf{H}_{0}^{\text {div }}(\Omega)$ and the matching pressure space $Q_{h} \subset Q$ of degree $k$, relative to $\mathbb{P}_{k}$ or $\mathbb{Q}_{k}$ on simplices or parallelograms/parallepipeds, respectively. The simplest choice is the Raviart-Thomas element [32] of order $k$. We assume that these pairs of spaces satisfy the following key property, like in the continuous case.

\section{Assumption 2.}

$$
\nabla \cdot \mathbf{V}_{h}=Q_{h}
$$

An immediate consequence of this assumption is that for each function $\mathbf{v} \in \mathbf{V}_{h}$, the condition $\left(\nabla \cdot \mathbf{v}, q_{h}\right)_{\Omega}=0$ for all $q_{h} \in Q_{h}$ implies that $\nabla \cdot \mathbf{v}=0$. Thus, we define the divergence-free subspace

$$
\stackrel{\circ}{\mathbf{V}}_{h}=\left\{\mathbf{v}_{h} \in \mathbf{V}_{h} \mid \nabla \cdot \mathbf{v}_{h}=0\right\} .
$$

The Stokes operator is discretized by a DG method. We shall not describe a particular DG method (see for example [4, 14, 27,33,34]), but just introduce some general tools and assumptions. A unit normal vector $\mathbf{n}_{F}$ is defined on each face $F$ of $\Gamma_{h}^{S}$; its direction can be chosen arbitrarily for each interior face, while it is outward for boundary faces. In the following, it will simply be denoted by $\mathbf{n}$. The jump of traces of a discontinuous function $\mathbf{v}$ across a face $F$ of $\Gamma_{i}^{S}$, in the direction of $\mathbf{n}_{F}$ is denoted by $\llbracket \mathbf{v} \rrbracket$. The jump on a boundary face is simply $\llbracket \mathbf{v} \rrbracket=\mathbf{v}$. Note that by the choice of spaces, the normal components of both jumps are zero, so that the jumps only act on the tangential components. The following DG-norm is used:

$$
\|\mathbf{v}\|_{1, h}=\left(\|\nabla \mathbf{v}\|_{\mathbb{T}_{h}^{S}}^{2}+\left\|\sigma_{h} \llbracket \mathbf{v} \rrbracket\right\|_{\Gamma_{h}^{S}}^{2}+\sum_{j=1}^{d-1}\left\|\gamma K^{-\frac{1}{4}} \mathbf{v} \cdot \tau^{j}\right\|_{\Gamma_{S D}}^{2}\right)^{\frac{1}{2}}
$$


where on an interior face $F$ between two mesh cells $T_{1}$ and $T_{2}$, we choose the weight $\sigma_{h}^{F}=\sqrt{\frac{1}{2}\left(\sigma_{h}^{F, 1}+\sigma_{h}^{F, 2}\right)}$. The parameter $\sigma_{h}^{F, i}$ has the form

$$
\sigma_{h}^{F, i}=\frac{\sigma}{h_{F, i}}
$$

with $\sigma>0$, independent of $h$, and $h_{F, i}$ the diameter of the cell $T_{i}$ measured perpendicular to $F$. On a face $F$ in $\Gamma_{b}^{S}$, where there is only a single cell $T_{1}$, we choose $\sigma_{h}^{F}=\sqrt{2 \sigma_{h}^{F, 1}}$ (see e.g. [27]). In the sequel, these weight functions are denoted $\sigma_{h}$. The norm of $\mathbf{V}_{h}$ is obtained by combining the $\mathbf{H}^{\text {div }}$ norm on $\Omega$ and the DG-norm on $\Omega_{S}$ :

$$
\|\mathbf{v}\|_{\mathbf{v}_{h}}=\left(\left\|K^{-\frac{1}{2}} \mathbf{v}\right\|_{\mathbf{L}^{2}\left(\Omega_{D}\right)}^{2}+\|\nabla \cdot \mathbf{v}\|_{L^{2}(\Omega)}^{2}+\|\mathbf{v}\|_{1, h}^{2}\right)^{\frac{1}{2}}
$$

Note that this is a norm owing to Poincaré's inequality for DG methods derived by Brenner in $[6,7]$.

Next, we introduce an approximation operator $R_{h} \in \mathcal{L}\left(\mathbf{V} \cap \mathbf{H}^{s}(\Omega) ; \mathbf{V}_{h}\right), s>0$, satisfying the following approximation properties.

Assumption 3. Let $k \geq 1$ be the degree of $Q_{h}$. Then, we assume the following properties of the operator $R_{h}$ : First, for any $s>0$ and $\mathbf{v} \in \mathbf{V} \cap \mathbf{H}^{s}(\Omega)$ there holds

$$
\left(\nabla \cdot R_{h}(\mathbf{v}), q_{h}\right)_{\Omega}=\left(\nabla \cdot \mathbf{v}, q_{h}\right)_{\Omega} \quad \forall q_{h} \in Q_{h},
$$

Then, for any cell $T \in \mathbb{T}_{h}$ and function $\mathbf{v} \in \mathbf{H}^{s}(T)$ with $0<s \leq k+1$ there holds

$$
\left\|R_{h}(\mathbf{v})-\mathbf{v}\right\|_{\mathbf{L}^{2}(T)} \leq C h_{T}^{s}|\mathbf{v}|_{\mathbf{H}^{s}(T)},
$$

and for $0 \leq s \leq k, 0 \leq t \leq s$, and $\mathbf{v} \in \mathbf{H}^{s+1}(T)$, we have

$$
\begin{aligned}
\left\|R_{h}(\mathbf{v})-\mathbf{v}\right\|_{\mathbf{H}^{t}(T)} & \leq C h_{T}^{s+1-t}|\mathbf{v}|_{\mathbf{H}^{s+1}(T)}, \\
\left\|\nabla \cdot\left(R_{h}(\mathbf{v})-\mathbf{v}\right)\right\|_{L^{2}(T)} & \leq C h_{T}^{s} \quad|\nabla \cdot \mathbf{v}|_{\mathbf{H}^{s}(T)}, \\
\left\|R_{h}(\mathbf{v})-\mathbf{v}\right\|_{\mathbf{L}^{2}(F)} & \leq C h_{T}^{s+\frac{1}{2}}|\mathbf{v}|_{\mathbf{H}^{s+1}(T)},
\end{aligned}
$$

where $F$ is any face of $T$. All constants $C$ are independent of $h, T$, or $F$.

Note that by definition, $R_{h}(\mathbf{v})$ is in $\mathbf{V}_{h}$; therefore

$$
R_{h}(\mathbf{v}) \cdot \mathbf{n}=0 \quad \text { on } \partial \Omega .
$$

For example, Assumption 3 is satisfied by the Raviart-Thomas interpolant $R_{h}$, see e.g. [23, 27]. For Navier-Stokes equations, the BDM pair was suggested in [12], the Raviart-Thomas pair in [13] (see also [38]).

An immediate consequence of this assumption is that for $s \leq k$ and for any $\mathbf{v} \in$ $\mathbf{H}^{s+1}(\Omega)$ there holds

$$
\left\|R_{h}(\mathbf{v})-\mathbf{v}\right\|_{1, h} \leq C h^{s}|\mathbf{v}|_{\mathbf{H}^{s+1}(\Omega)} .
$$

It yields stability in the $\mathbf{V}_{h}$ norm over the whole region and this is sufficient to establish the following uniform inf-sup condition: There exists a constant $\beta^{\star}>0$, independent of $h$, such that

$$
\forall q_{h} \in Q_{h}, \sup _{\mathbf{v}_{h} \in \mathbf{V}_{h}} \frac{b\left(\mathbf{v}_{h}, q_{h}\right)}{\left\|\mathbf{v}_{h}\right\|_{\mathbf{v}_{h}}} \geq \beta^{\star}\left\|q_{h}\right\|_{L^{2}(\Omega)} .
$$

This follows easily from (10), (21), (24) and (27) with $s=0$. 
2.2.2. The discontinuous Galerkin bilinear form. We use a discrete bilinear form $a_{S, h}(\cdot, \cdot)$ based on a discontinuous Galerkin method in the Stokes subdomain and formally define for all sufficiently smooth $\mathbf{u}, \mathbf{v}$

$$
a_{h}(\mathbf{u}, \mathbf{v})=a_{D}(\mathbf{u}, \mathbf{v})+a_{I}(\mathbf{u}, \mathbf{v})+a_{S, h}(\mathbf{u}, \mathbf{v}) .
$$

Then, for solution pairs $(\mathbf{u}, p) \in \mathbf{V} \times Q$ of (7) and $\left(\mathbf{u}^{*}, p^{*}\right) \in \mathbf{V} \times Q$ of (12), and for any test function $\mathbf{v} \in \mathbf{V}+\mathbf{V}_{h}$, we define the primal and dual residual operators

$$
\begin{aligned}
\operatorname{Res}(\mathbf{u}, p ; \mathbf{v}) & =a_{h}(\mathbf{u}, \mathbf{v})+b(\mathbf{v}, p)-(\mathbf{f}, \mathbf{v})_{\Omega}, \\
\operatorname{Res}^{*}\left(\mathbf{v} ; \mathbf{u}^{*}, p^{*}\right) & =a_{h}\left(\mathbf{v}, \mathbf{u}^{*}\right)+b\left(\mathbf{v}, p^{*}\right)-(\boldsymbol{\psi}, \mathbf{v})_{\Omega_{S}} .
\end{aligned}
$$

This definition is motivated by the next lemma.

Lemma 2. Let $\mathbf{f} \in \mathbf{L}^{2}(\Omega)$ and let the solution (u,p) of (2), (3) satisfy $p_{D} \in H^{1}\left(\Omega_{D}\right)$, $\mathbf{u} \in \mathbf{V}, \mathbf{u}_{S} \in \mathbf{H}^{s}(\Omega)$, and $p_{S} \in H^{s-1}\left(\Omega_{S}\right)$ for some real number $s>\frac{3}{2}$. Then we have for all $\mathbf{v} \in \mathbf{V}+\mathbf{V}_{h}$ :

$$
(\mathbf{f}, \mathbf{v})_{\Omega}=a_{h}(\mathbf{u}, \mathbf{v})-a_{S, h}(\mathbf{u}, \mathbf{v})+b(\mathbf{v}, p)+2 \nu\left((\varepsilon(\mathbf{u}), \varepsilon(\mathbf{v}))_{\mathbb{T}_{h}^{S}}-\langle\varepsilon(\mathbf{u}) \mathbf{n}, \llbracket \mathbf{v} \rrbracket\rangle_{\Gamma_{h}^{S}}\right) .
$$

Proof. We have

$$
(\mathbf{f}, \mathbf{v})_{\Omega}=(-\nabla \cdot(2 \nu \varepsilon(\mathbf{u}))+\nabla p, \mathbf{v})_{\Omega_{S}}+\left(K^{-1} \mathbf{u}+\nabla p, \mathbf{v}\right)_{\Omega_{D}} .
$$

The regularity of the functions $\mathbf{f}, \mathbf{u}_{D}$, and $p_{D}$, Green's formula, the value of $\mathbf{v}$ on $\Gamma_{D}$ and the orientation of the normal on $\Gamma_{S D}$ easily give

$$
\left(K^{-1} \mathbf{u}+\nabla p, \mathbf{v}\right)_{\Omega_{D}}=\left(K^{-1} \mathbf{u}, \mathbf{v}\right)_{\Omega_{D}}-(p, \nabla \cdot \mathbf{v})_{\Omega_{D}}-\left\langle p_{D}, \mathbf{v} \cdot \mathbf{n}\right\rangle_{\Gamma_{S D}}
$$

In particular, as $\mathbf{v} \cdot \mathbf{n}=0$ on $\Gamma_{D}$, the work of Galvis and Sarkis in [16] implies that the last duality makes sense. Next, the regularity assumptions on $\mathbf{u}_{S}$ and $p_{S}$ imply that the traces of both $\varepsilon\left(\mathbf{u}_{S}\right)$ and $p_{S}$ on elements' faces belong to $H^{t}$ with $t=s-\frac{3}{2}>0$, hence are in $L^{2}$. Then the regularity of $\mathbf{f}$ and $\mathbf{v}$, Green's formula, and the value of $\mathbf{v}$ on $\Gamma_{S}$ yield

$$
\begin{aligned}
(-\nabla \cdot(2 \nu \varepsilon(\mathbf{u}))+\nabla p, \mathbf{v})_{\Omega_{S}}=2 \nu\left((\varepsilon(\mathbf{u}), \varepsilon(\mathbf{v}))_{\mathbb{T}_{h}^{S}}\right. & \left.-\sum_{T \in \mathbb{T}_{h}^{S}}\left\langle\varepsilon(\mathbf{u}) \mathbf{n}_{T}, \mathbf{v}\right\rangle_{\partial T}\right) \\
& -(p, \nabla \cdot \mathbf{v})_{\Omega_{S}}+\left\langle p_{S}, \mathbf{v} \cdot \mathbf{n}\right\rangle_{\Gamma_{S D}},
\end{aligned}
$$

where $\mathbf{n}_{T}$ denotes the unit normal vector on $\partial T$, exterior to $T$. Again, the regularity assumption on $\mathbf{u}_{S}$ imply that

$$
\sum_{T \in \mathbb{T}_{h}^{S}}\left\langle\varepsilon(\mathbf{u}) \mathbf{n}_{T}, \mathbf{v}\right\rangle_{\partial T}=\langle\varepsilon(\mathbf{u}) \mathbf{n}, \llbracket \mathbf{v} \rrbracket\rangle_{\Gamma_{h}^{S}}+\left\langle\varepsilon\left(\mathbf{u}_{S}\right) \mathbf{n}, \mathbf{v}\right\rangle_{\Gamma_{S D}}
$$

Collecting these two equalities, we obtain

$$
\begin{aligned}
(\mathbf{f}, \mathbf{v})_{\Omega}=\left(K^{-1} \mathbf{u}, \mathbf{v}\right)_{\Omega_{D}}-(p, \nabla \cdot \mathbf{v})_{\Omega}+2 \nu(\varepsilon(\mathbf{u}), \varepsilon(\mathbf{v}))_{\mathbb{T}_{h}^{S}} & \\
& -2 \nu\langle\varepsilon(\mathbf{u}) \mathbf{n}, \llbracket \mathbf{v} \rrbracket\rangle_{\Gamma_{h}^{S}}+\left\langle-2 \nu \varepsilon\left(\mathbf{u}_{S}\right) \mathbf{n}+\left(p_{S}-p_{D}\right) \mathbf{n}, \mathbf{v}\right\rangle_{\Gamma_{S D}} .
\end{aligned}
$$

Then the boundary conditions ( $3 b)$ and ( $3 c)$ on the interface $\Gamma_{S D}$ give

$$
\left\langle-2 \nu \varepsilon\left(\mathbf{u}_{S}\right) \mathbf{n}+\left(p_{S}-p_{D}\right) \mathbf{n}, \mathbf{v}\right\rangle_{\Gamma_{S D}}=a_{I}(\mathbf{u}, \mathbf{v}),
$$

and (30) follows immediately from the definition of $a_{h}(\cdot, \cdot)$.

From this lemma, we deduce immediately the following corollary. 
Corollary 3. Under the assumption of Lemma 2, the residuals can be expressed as:

$$
\begin{gathered}
\operatorname{Res}(\mathbf{u}, p ; \mathbf{v})=a_{S, h}(\mathbf{u}, \mathbf{v})-2 \nu(\varepsilon(\mathbf{u}), \varepsilon(\mathbf{v}))_{\mathbb{T}_{h}^{S}}+2 \nu\langle\varepsilon(\mathbf{u}) \mathbf{n}, \llbracket \mathbf{v} \rrbracket\rangle_{\Gamma_{h}^{S}}, \\
\operatorname{Res}^{*}\left(\mathbf{v} ; \mathbf{u}^{*}, p^{*}\right)=a_{S, h}\left(\mathbf{v}, \mathbf{u}^{*}\right)-2 \nu\left(\varepsilon\left(\mathbf{u}^{*}\right), \varepsilon(\mathbf{v})\right)_{\mathbb{T}_{h}^{S}}+2 \nu\left\langle\varepsilon\left(\mathbf{u}^{*}\right) \mathbf{n}, \llbracket \mathbf{v} \rrbracket\right\rangle_{\Gamma_{h}^{S}} .
\end{gathered}
$$

Thus none of the residuals depend on $p$ or $p^{*}$ and from now on we denote them by $\operatorname{Res}(\mathbf{u} ; \mathbf{v})$ and $\operatorname{Res}^{*}\left(\mathbf{v} ; \mathbf{u}^{*}\right)$.

Now, instead of specifying $a_{S, h}(\cdot, \cdot)$, we make some generic assumptions.

Assumption 4. The bilinear form $a_{S, h}(\mathbf{u}, \mathbf{v})$ of the DG method chosen in the Stokes subdomain $\Omega_{S}$ has the following four properties:

(a) Stability in the DG norm: There exists a constant $\alpha^{\star}$ independent of $h$, such that for any functions $\mathbf{u}_{h}, \mathbf{v}_{h} \in \mathbf{V}_{h}$ there holds

$$
a_{S, h}\left(\mathbf{u}_{h}, \mathbf{u}_{h}\right) \geq \alpha^{\star}\left\|\mathbf{u}_{h}\right\|_{1, h}^{2} .
$$

(b) Boundedness in the DG norm: There exists a constant $M^{\star}$, independent of $h$, such that for any functions $\mathbf{u}, \mathbf{v} \in \mathbf{V}+\mathbf{V}_{h}$ there holds

$$
a_{S, h}(\mathbf{u}, \mathbf{v}) \leq M^{\star}\|\mathbf{u}\|_{1, h}\|\mathbf{v}\|_{1, h} .
$$

(c) Consistency: With the assumptions and notation of Lemma 2 regarding the solution $(\mathbf{u}, p)$ of (7), there exists a constant $C$, independent of $h$, such that for all $\mathbf{v} \in \mathbf{V}+\mathbf{V}_{h}$

$$
|\operatorname{Res}(\mathbf{u} ; \mathbf{v})| \leq C h^{s-1}|\mathbf{u}|_{H^{s}\left(\Omega_{S}\right)}\left\|\sigma_{h} \llbracket \mathbf{v} \rrbracket\right\|_{\Gamma_{h}^{S}} .
$$

(d) Adjoint consistency: With the assumptions and notation of Lemma 2 regarding the solution $\left(\mathbf{u}^{*}, p^{*}\right)$ of (12), there exists a constant $C^{*}$, independent of $h$, such that for all $\mathbf{v} \in \mathbf{V}+\mathbf{V}_{h}$

$$
\left|\operatorname{Res}^{*}\left(\mathbf{v} ; \mathbf{u}^{*}\right)\right| \leq C^{\star} h^{s-1}\left|\mathbf{u}^{*}\right|_{H^{s}\left(\Omega_{S}\right)}\left\|\sigma_{h} \llbracket \mathbf{v} \rrbracket\right\|_{\Gamma_{h}^{S}} .
$$

The assumptions imposed here follow those in [37], and the application to various DG methods is explained there. As an example, we reproduce the form of the interior penalty method for which (34) holds. To this end, we first introduce the lifting operator $\mathcal{L}$ from $\mathbf{V}+$ $\mathbf{V}_{h}$ into the space $\Sigma_{h}=\left\{\tau \in L^{2}\left(\Omega ; \mathbb{R}^{d \times d}\right)\left|\forall T \in \mathbb{T}_{h}: \tau_{i j}\right|_{T} \in \mathcal{P}(T)\right\}$ of discontinuous $d \times d$ tensor functions with components in the space $\mathcal{P}(T)$ on each cell $T$. The space $\mathcal{P}(T)$ is a possibly mapped polynomial space on $T$ such that $\varepsilon\left(v_{T}\right) \in \mathcal{P}(T)$ for all $v_{T}$ in the discrete velocity space on $T$.

$$
(\mathcal{L} \mathbf{v}, \tau)_{\mathbb{T}_{h}^{S}}=\langle\llbracket \mathbf{v} \rrbracket,\{\{\tau \mathbf{n}\}\}\rangle_{\Gamma_{h}^{S}} \quad \forall \tau \in \Sigma_{h},
$$

where $\{\{\tau \mathbf{n}\}\}$ denotes the pointwise mean value of $\tau \mathbf{n}$ on the two adjacent cells, or the value of $\tau \mathbf{n}$ itself on a boundary face and where $\left.\mathbf{n}\right|_{F}=\mathbf{n}_{F}$. Then, the symmetric interior penalty discretization of the Stokes problem is

$$
a_{S, h}(\mathbf{u}, \mathbf{v})=2 \nu\left((\varepsilon(\mathbf{u}), \varepsilon(\mathbf{v}))_{\mathbb{T}_{h}^{S}}-(\mathcal{L} \mathbf{u}, \varepsilon(\mathbf{v}))_{\mathbb{T}_{h}^{S}}-(\varepsilon(\mathbf{u}), \mathcal{L} \mathbf{v})_{\mathbb{T}_{h}^{S}}+\left\langle\sigma_{h}^{2} \llbracket \mathbf{u} \rrbracket, \llbracket \mathbf{v} \rrbracket\right\rangle_{\Gamma_{h}^{S}}\right),
$$


which, restricted to the discrete space $\mathbf{V}_{h}$, is equivalent to the usual form

$$
\begin{aligned}
a_{S, h}(\mathbf{u}, \mathbf{v})=2 \nu\left((\varepsilon(\mathbf{u}), \varepsilon(\mathbf{v}))_{\mathbb{T}_{h}^{S}}+\left\langle\sigma_{h}^{2} \llbracket \mathbf{u} \rrbracket, \llbracket \mathbf{v} \rrbracket\right\rangle_{\Gamma_{h}^{S}}\right. & \\
& -\left\langle\llbracket \mathbf{u} \rrbracket,\{\{\varepsilon(\mathbf{v}) \mathbf{n}\}\rangle_{\Gamma_{h}^{S}}-\left\langle\{\{\varepsilon(\mathbf{u}) \mathbf{n}\}, \llbracket \mathbf{v} \rrbracket\rangle_{\Gamma_{h}^{S}}\right) .\right.
\end{aligned}
$$

Following [37], the lifting version of the LDG discretization (see [14]) is obtained as

$$
a_{S, h}(\mathbf{u}, \mathbf{v})=2 \nu\left((\varepsilon(\mathbf{u})-\mathcal{L} \mathbf{u}, \varepsilon(\mathbf{v})-\mathcal{L} \mathbf{v})_{\mathbb{T}_{h}^{S}}+\left\langle\sigma_{h}^{2} \llbracket \mathbf{u} \rrbracket, \llbracket \mathbf{v} \rrbracket\right\rangle_{\Gamma_{h}^{S}}\right) .
$$

Since it is shown in [37] that the operator $\mathcal{L}$ is bounded on $\mathbf{V}+\mathbf{V}_{h}$ with respect to the norm $\|\cdot\|_{\mathbf{V}_{h}}$, we obtain (34). By applying Korn's inequality [8] we derive also (33). In the case of the symmetric interior penalty method, the penalty parameter is assumed to be sufficiently large.

While the lifting form of the methods enables us to use boundedness and ellipticity estimates in the natural norm $\|\cdot\|_{1, h}$, it is not consistent. Nevertheless, the two conditions (35) and (36) which are a refinement of [37, Proposition 8.1] hold, as the following lemma shows. Note also that while we do not require symmetry of $a_{S, h}(\cdot, \cdot)$, it implies the equivalence of (35) and (36).

Lemma 4. The primal and dual residuals of the discrete bilinear forms in equations (37) and (38) satisfy the estimates with constants $C$ independent of $h$

$$
\begin{aligned}
|\operatorname{Res}(\mathbf{u} ; \mathbf{v})| & \leq C h^{s-1}|\mathbf{u}|_{H^{s}\left(\Omega_{S}\right)}\left\|\sigma_{h} \llbracket \mathbf{v} \rrbracket\right\|_{\Gamma_{h}^{S}} \\
\left|\operatorname{Res}^{*}(\mathbf{u} ; \mathbf{v})\right| & \leq C h^{s-1}|\mathbf{u}|_{H^{s}\left(\Omega_{S}\right)}\left\|\sigma_{h} \llbracket \mathbf{v} \rrbracket\right\|_{\Gamma_{h}^{S}},
\end{aligned}
$$

under the regularity assumptions of Lemma 2.

Proof. First, we note that both forms (37) and (38) are symmetric, and thus it suffices to show only one of the inequalities. Next, we observe that the jump $\llbracket \mathbf{u} \rrbracket=\mathbf{0}$ and thus $\mathcal{L} \mathbf{u}=0$. This implies for both forms

$$
a_{S, h}(\mathbf{u}, \mathbf{v})=2 \nu\left((\varepsilon(\mathbf{u}), \varepsilon(\mathbf{v}))_{\mathbb{T}_{h}^{S}}-(\varepsilon(\mathbf{u}), \mathcal{L} \mathbf{v})_{\mathbb{T}_{h}^{S}}\right) .
$$

Hence (31) and the regularity of $\mathbf{u}$ give

$$
\operatorname{Res}(\mathbf{u} ; \mathbf{v})=2 \nu\left(\langle\varepsilon(\mathbf{u}) \mathbf{n}, \llbracket \mathbf{v} \rrbracket\rangle_{\Gamma_{h}^{S}}-(\varepsilon(\mathbf{u}), \mathcal{L} \mathbf{v})_{\mathbb{T}_{h}^{S}}\right)=2 \nu\left(\langle\{\{\varepsilon(\mathbf{u}) \cdot \mathbf{n}\}\}, \llbracket \mathbf{v} \rrbracket\rangle_{\Gamma_{h}^{S}}-(\varepsilon(\mathbf{u}), \mathcal{L} \mathbf{v})_{\mathbb{T}_{h}^{S}}\right) .
$$

Since $\mathcal{L} \mathbf{v}$ is in $\Sigma_{h}$, we may insert the $L^{2}$ projection $\Pi_{\Sigma_{h}}$ into $\Sigma_{h}$ in the term on the right. Then, using the definition of the lifting operator, we obtain

$$
\operatorname{Res}(\mathbf{u} ; \mathbf{v})=2 \nu\left\langle\frac{1}{\sigma_{h}}\left\{\left\{\varepsilon(\mathbf{u})-\Pi_{\Sigma_{h}} \varepsilon(\mathbf{u})\right\}, \sigma_{h} \llbracket \mathbf{v} \rrbracket\right\rangle_{\Gamma_{h}^{S}} .\right.
$$

Applying a trace inequality on the reference element and standard approximation results, we obtain

$$
|\operatorname{Res}(\mathbf{u} ; \mathbf{v})| \leq C h^{s-1}|\mathbf{u}|_{H^{s}(\Omega)}\left\|\sigma_{h} \llbracket \mathbf{v} \rrbracket\right\|_{\Gamma_{h}^{S}} .
$$

The discrete formulation of the problem (2)-(3) then reads: find $\left(\mathbf{u}_{h}, p_{h}\right) \in \mathbf{V}_{h} \times Q_{h}$ such that

$$
\begin{aligned}
a_{h}\left(\mathbf{u}_{h}, \mathbf{v}_{h}\right)+b\left(\mathbf{v}_{h}, p_{h}\right) & =\left(\mathbf{f}, \mathbf{v}_{h}\right)_{\Omega}, & & \forall \mathbf{v}_{h} \in \mathbf{V}_{h}, \\
b\left(\mathbf{u}_{h}, q_{h}\right) & =-\left(g, q_{h}\right)_{\Omega}, & & \forall q_{h} \in Q_{h} .
\end{aligned}
$$

Under the above assumptions, it is easy to prove that (39) has exactly one solution [28]. 


\section{ERRor ANALYSIS}

The error analysis is conducted in several steps. First, we recall the energy norm estimate from [28]. The problem with this estimate is that it is suboptimal in the Darcy subdomain $\Omega_{D}$, and that it is not possible to improve it there by a simple duality argument. Nevertheless, such a duality argument is applied in the Stokes subdomain $\Omega_{S}$ in subsection 3.2. In conjunction with the divergence approximation of the Raviart-Thomas element, this yields a sharp estimate on the interface $\Gamma_{S D}$ in subsection 3.3, which in turn serves as an input to the optimal estimate for the Darcy velocity in subsection 3.4. The outcome of these steps will be our main result:

Theorem 5. Let Assumptions 1-4 hold. Assume further, that the solution $\mathbf{u}$ of the weak formulation (7) is in $\mathbf{H}^{s}(\Omega)$ for some $\left.\left.s \in\right] \frac{3}{2}, k+1\right]$ with $k \geq 1$ from Assumption 3 and $\left.\alpha \in] \frac{1}{2}, 1\right]$ determined by Assumption 1. Suppose $g$ from (2c) belongs to $H^{s-1+\alpha}(\Omega)$. Then, the solution $\mathbf{u}_{h} \in \mathbf{V}_{h}$ of (39) satisfies the $L^{2}$ error estimate

$$
\left\|\mathbf{u}-\mathbf{u}_{h}\right\|_{\mathbf{L}^{2}(\Omega)} \leq C h^{s+\alpha-1}\left(|\mathbf{u}|_{\mathbf{H}^{s}(\Omega)}+|g|_{H^{s-1+\alpha}(\Omega)}\right)
$$

with a constant $C$ independent of $h$ and $\mathbf{u}$. A direct corollary is the optimal rate $h^{s}$ in the case where the dual problem has full regularity $(\alpha=1)$.

Proof. The proof is an immediate consequence of Theorems 9 and 12 below.

In [28, Table 3], convergence rates in $L^{2}(\Omega)$ had already been presented. There, we obtained for a very regular solution convergence rates $h^{k+1}$ for $u_{S}$ and $u_{D}$ for $k \leq 3$; for higher order elements, the corner singularities were too strong. We refer to this publication for details.

3.1. First error analysis. Using standard stability and approximation arguments, we easily establish the following variant of a result that was proved in [28]. Note that $k \geq 1$ is required, since the strong norm in the Stokes subdomain does not allow for convergence of the lowest order Raviart-Thomas element.

Proposition 6. Let assumptions 2-4 hold. Assume further, that the solution $\mathbf{u}$ of the weak formulation (7) is in $\mathbf{H}^{s}(\Omega)$ for some $\left.\left.s \in\right] \frac{3}{2}, k+1\right]$. Then, the solutions $\mathbf{u}$ and $\mathbf{u}_{h}$ of the coupled systems (7) and (39) have the energy error estimate

$$
\left\|\mathbf{u}-\mathbf{u}_{h}\right\|_{\mathbf{v}_{h}} \leq C h^{s-1}|\mathbf{u}|_{\mathbf{H}^{s}(\Omega)},
$$

with a constant $C$ independent of $h$ and $\mathbf{u}$.

Proof. We briefly recall the proof for the readers' convenience. From (7), (29a), and (39), we have:

$$
\begin{aligned}
a_{h}\left(\mathbf{u}-\mathbf{u}_{h}, \mathbf{v}_{h}\right)+b\left(\mathbf{v}_{h}, p-p_{h}\right) & =\operatorname{Res}\left(\mathbf{u} ; \mathbf{v}_{h}\right), & & \forall \mathbf{v}_{h} \in \mathbf{V}_{h}, \\
b\left(\mathbf{u}-\mathbf{u}_{h}, q_{h}\right) & =0, & & \forall q_{h} \in Q_{h} .
\end{aligned}
$$

Inserting $R_{h}(\mathbf{u})$, this gives

$$
\begin{aligned}
& a_{h}\left(R_{h}(\mathbf{u})-\mathbf{u}_{h}, \mathbf{v}_{h}\right)+b\left(\mathbf{v}_{h}, p-p_{h}\right)=\operatorname{Res}\left(\mathbf{u} ; \mathbf{v}_{h}\right)-a_{h}\left(\mathbf{u}-R_{h}(\mathbf{u}), \mathbf{v}_{h}\right), \quad \forall \mathbf{v}_{h} \in \mathbf{V}_{h}, \\
& b\left(R_{h}(\mathbf{u})-\mathbf{u}_{h}, q_{h}\right) \quad=\quad 0, \quad \forall q_{h} \in Q_{h} \text {. }
\end{aligned}
$$

Owing to (21), this last equation is due to the fact that $b\left(\mathbf{u}-R_{h}(\mathbf{u}), q_{h}\right)=0$ for all $q_{h} \in Q_{h}$; according to (17), it implies that $\nabla \cdot\left(\mathbf{u}_{h}-R_{h}(\mathbf{u})\right)=0$. Then (40) easily follows by choosing $\mathbf{v}_{h}=R_{h}(\mathbf{u})-\mathbf{u}_{h} \in \stackrel{\circ}{\mathbf{V}}_{h}$ in (42) and using (33), (34), (35), and the approximation properties of $R_{h}$ (22)-(25). 
Note that (17) and (41) yield, if we denote by $\Pi_{Q_{h}}$ the $L^{2}$ projection onto $Q_{h}$ :

Proposition 7. The divergence of the discrete solution $\mathbf{u}_{h}$ is the $L^{2}$ projection of the divergence of $\mathbf{u}$ onto the discrete pressure space $Q_{h}$, or $\nabla \cdot \mathbf{u}_{h}=\Pi_{Q_{h}} \nabla \cdot \mathbf{u}=\Pi_{Q_{h}} g$.

This implies the following result (see for instance [10]).

Corollary 8. Let $\mathbf{u}$ and $\mathbf{u}_{h}$ be the solutions to (7) and (39), respectively. Suppose $g$ from (2c) belongs to $H^{s}(\Omega)$ with $s \in[0, k+1]$; then

$$
\left\|\nabla \cdot\left(\mathbf{u}-\mathbf{u}_{h}\right)\right\|_{L^{2}(\Omega)} \leq C h^{s}|g|_{H^{s}(\Omega)} .
$$

3.2. Sharper error estimate in the Stokes subdomain. The energy norm in the Stokes subdomain $\Omega_{S}$ involves the derivatives of $\mathbf{u}_{S}$. Thus, in this subdomain we can employ a duality argument of Aubin and Nitsche to obtain the following theorem:

Theorem 9. Let Assumptions $1-4$ hold with the constant $\left.\alpha \in] \frac{1}{2}, 1\right]$ determined by Assumption 1. Let $\mathbf{u} \in \mathbf{H}^{s}(\Omega)$ for $\left.\left.s \in\right] \frac{3}{2}, k+1\right]$ be the solution of (7) and let $\mathbf{u}_{h}$ be the solution to (39). Then, the following error estimate holds

$$
\left\|\mathbf{u}-\mathbf{u}_{h}\right\|_{\mathbf{L}^{2}\left(\Omega_{S}\right)} \leq C h^{s-1+\alpha}|\mathbf{u}|_{H^{s}(\Omega)} .
$$

Proof. Let $\left(\mathbf{u}^{*}, p^{*}\right)$ be a solution of (12) with $\psi=\chi\left(\Omega_{S}\right)\left(\mathbf{u}-\mathbf{u}_{h}\right)$, where $\chi\left(\Omega_{S}\right)$ is the indicator function of $\Omega_{S}$. It follows from (29b) with $\left(\mathbf{u}^{*}, p^{*}\right)$ and $\mathbf{f}=\chi\left(\Omega_{S}\right)\left(\mathbf{u}-\mathbf{u}_{h}\right)$ that for all $\mathbf{v} \in \mathbf{V}+\mathbf{V}_{h}$

$$
\left(\mathbf{v}, \mathbf{u}-\mathbf{u}_{h}\right)_{\Omega_{S}}=a_{h}\left(\mathbf{v}, \mathbf{u}^{*}\right)+b\left(\mathbf{v}, p^{*}\right)-\operatorname{Res}^{*}\left(\mathbf{v} ; \mathbf{u}^{*}\right) .
$$

On one hand, the choice $\mathbf{v}=\mathbf{u}-\mathbf{u}_{h}$ gives

$$
\left\|\mathbf{u}-\mathbf{u}_{h}\right\|_{L^{2}\left(\Omega_{S}\right)}^{2}=a_{h}\left(\mathbf{u}-\mathbf{u}_{h}, \mathbf{u}^{*}\right)+b\left(\mathbf{u}-\mathbf{u}_{h}, p^{*}\right)-\operatorname{Res}^{*}\left(\mathbf{u}-\mathbf{u}_{h} ; \mathbf{u}^{*}\right) .
$$

On the other hand, the error equation (41) with $\mathbf{v}_{h}=R_{h} \mathbf{u}^{*} \in \mathbf{V}_{h}$ implies

$$
a_{h}\left(\mathbf{u}-\mathbf{u}_{h}, R_{h} \mathbf{u}^{*}\right)+b\left(R_{h} \mathbf{u}^{*}, p-p_{h}\right)=\operatorname{Res}\left(\mathbf{u} ; R_{h} \mathbf{u}^{*}\right) .
$$

But $b\left(R_{h} \mathbf{u}^{*}, p-p_{h}\right)=0$ because $\nabla \cdot R_{h} \mathbf{u}^{*}=0$; this follows from the fact that $\nabla \cdot \mathbf{u}^{*}=0$, Assumption 2, and property (21) of $R_{h}$. Therefore combining with (45), we obtain for all $q_{h} \in Q_{h}$

$$
\begin{aligned}
\left\|\mathbf{u}-\mathbf{u}_{h}\right\|_{L^{2}\left(\Omega_{S}\right)}^{2}=a_{h}\left(\mathbf{u}-\mathbf{u}_{h}, \mathbf{u}^{*}-R_{h} \mathbf{u}^{*}\right)+b\left(\mathbf{u}-\mathbf{u}_{h}, p^{*}-q_{h}\right) & -\operatorname{Res}^{*}\left(\mathbf{u}-\mathbf{u}_{h} ; \mathbf{u}^{*}\right) \\
& +\operatorname{Res}\left(\mathbf{u} ; R_{h} \mathbf{u}^{*}\right) .
\end{aligned}
$$

This yields

$$
\begin{aligned}
\left\|\mathbf{u}-\mathbf{u}_{h}\right\|_{L^{2}\left(\Omega_{S}\right)}^{2} \leq\left|a_{h}\left(\mathbf{u}-\mathbf{u}_{h}, \mathbf{u}^{*}-R_{h} \mathbf{u}^{*}\right)\right| & +\left|b\left(\mathbf{u}-\mathbf{u}_{h}, p^{*}-q_{h}\right)\right| \\
& +\left|\operatorname{Res}^{*}\left(\mathbf{u}-\mathbf{u}_{h} ; \mathbf{u}^{*}\right)\right|+\left|\operatorname{Res}\left(\mathbf{u} ; R_{h} \mathbf{u}^{*}\right)\right| .
\end{aligned}
$$

The first term on the right-hand side is bounded as follows:

$$
\begin{aligned}
& a_{h}\left(\mathbf{u}-\mathbf{u}_{h}, \mathbf{u}^{*}-R_{h}\left(\mathbf{u}^{*}\right)\right)=a_{S, h}\left(\mathbf{u}_{S}-\mathbf{u}_{S h}, \mathbf{u}_{S}^{*}-R_{h}\left(\mathbf{u}_{S}^{*}\right)\right) \\
& \quad+a_{D}\left(\mathbf{u}_{D}-\mathbf{u}_{D h}, \mathbf{u}_{D}^{*}-R_{h}\left(\mathbf{u}_{D}^{*}\right)\right)+a_{I}\left(\mathbf{u}_{S}-\mathbf{u}_{S h}, \mathbf{u}_{S}^{*}-R_{h}\left(\mathbf{u}_{S}^{*}\right)\right) \\
& \leq C h^{\alpha}\left|\mathbf{u}_{S}^{*}\right|_{H^{1+\alpha}\left(\Omega_{S}\right)}\left\|\mathbf{u}_{S}-\mathbf{u}_{S h}\right\|_{1, h}+C h^{\alpha}\left|\mathbf{u}_{D}^{*}\right|_{H^{\alpha}\left(\Omega_{D}\right)}\left\|K^{-\frac{1}{2}}\left(\mathbf{u}_{D}-\mathbf{u}_{D h}\right)\right\|_{\mathbf{L}^{2}\left(\Omega_{D}\right)} \\
& \quad+C h^{\alpha+\frac{1}{2}}\left|\mathbf{u}_{S}^{*}\right|_{H^{1+\alpha}\left(\Omega_{S}\right)}\left(\sum_{j=1}^{d-1}\left\|\gamma K^{-\frac{1}{4}}\left(\mathbf{u}_{S}-\mathbf{u}_{S h}\right) \cdot \boldsymbol{\tau}^{j}\right\|_{L^{2}\left(\Gamma_{S D}\right)}^{2}\right)^{\frac{1}{2}}
\end{aligned}
$$


from the approximation properties (22), (25), and (27) of $R_{h}$. For the second term, we pick $q_{h}=\Pi_{Q_{h}} p^{*}:$

$$
\begin{gathered}
b\left(\mathbf{u}-\mathbf{u}_{h}, p^{*}-\Pi_{Q_{h}} p^{*}\right)=-\left(\nabla \cdot\left(\mathbf{u}_{S}-\mathbf{u}_{S h}\right), p_{S}^{*}-\Pi_{Q_{h}} p_{S}^{*}\right)_{\Omega_{S}} \\
-\left(\nabla \cdot\left(\mathbf{u}_{D}-\mathbf{u}_{D h}\right), p_{D}^{*}-\Pi_{Q_{h}} p_{D}^{*}\right)_{\Omega_{D}} .
\end{gathered}
$$

From the approximation properties of $Q_{h}$, we readily derive:

$$
\left|b\left(\mathbf{u}-\mathbf{u}_{h}, p^{*}-\Pi_{Q_{h}} p^{*}\right)\right| \leq C h^{\alpha}\left\|\nabla \cdot\left(\mathbf{u}_{S}-\mathbf{u}_{S h}\right)\right\|_{L^{2}(\Omega)}\left(\left|p^{*}\right|_{H^{\alpha}\left(\Omega_{S}\right)}+\left|p^{*}\right|_{H^{\alpha}\left(\Omega_{D}\right)}\right) .
$$

The adjoint residual term $\left|\operatorname{Res}^{*}\left(\mathbf{u}-\mathbf{u}_{h} ; \mathbf{u}^{*}\right)\right|$ can be estimated by the adjoint consistency estimate (36):

$$
\left|\operatorname{Res}^{*}\left(\mathbf{u}^{*} ; \mathbf{u}-\mathbf{u}_{h}\right)\right| \leq C h^{\alpha}\left|\mathbf{u}^{*}\right|_{H^{1+\alpha}\left(\Omega_{S}\right)}\left\|\sigma_{h} \llbracket \mathbf{u}-\mathbf{u}_{h} \rrbracket\right\|_{\Gamma_{h}^{S}} .
$$

For the primal residual, in view of the regularity of $\mathbf{u}^{*}$ and its boundary value on $\Gamma_{h}^{S}$, we observe that

$$
\begin{aligned}
\left|\operatorname{Res}\left(\mathbf{u} ; R_{h}\left(\mathbf{u}^{*}\right)\right)\right| \leq C h^{s-1}|\mathbf{u}|_{H^{s}\left(\Omega_{S}\right)} \| \sigma_{h} \llbracket u^{*} & -R_{h}\left(\mathbf{u}^{*}\right) \rrbracket \|_{\Gamma_{h}^{S}} \\
& \leq C h^{s+\alpha-1}|\mathbf{u}|_{H^{s}\left(\Omega_{S}\right)}\left|\mathbf{u}^{*}\right|_{H^{1+\alpha}\left(\Omega_{S}\right)} .
\end{aligned}
$$

Finally, a combination of the bounds above gives:

$$
\begin{gathered}
\left\|\mathbf{u}-\mathbf{u}_{h}\right\|_{\mathbf{L}^{2}\left(\Omega_{S}\right)} \leq C h^{\alpha}\left(\left\|\mathbf{u}_{S}-\mathbf{u}_{S h}\right\|_{1, h}+\left\|\mathbf{u}_{D}-\mathbf{u}_{D h}\right\|_{\mathbf{L}^{2}\left(\Omega_{D}\right)}\right. \\
+h^{\frac{1}{2}}\left(\sum_{j=1}^{d-1}\left\|\gamma K^{-\frac{1}{4}}\left(\mathbf{u}_{S}-\mathbf{u}_{S h}\right) \cdot \boldsymbol{\tau}^{j}\right\|_{L^{2}\left(\Gamma_{S D}\right)}^{2}\right)^{\frac{1}{2}} \\
\left.+\left\|\nabla \cdot\left(\mathbf{u}-\mathbf{u}_{h}\right)\right\|_{L^{2}(\Omega)}+h^{s-1}|\mathbf{u}|_{H^{s}\left(\Omega_{S}\right)}\right) .
\end{gathered}
$$

We now conclude by using (40).

3.3. Estimates on the interface. Since $\mathbf{u}_{S}-\mathbf{u}_{S h}$ belongs to $\mathbf{H}^{\text {div }}\left(\Omega_{S}\right)$, its normal trace satisfies (cf. [19])

$$
\left\|\left(\mathbf{u}_{S}-\mathbf{u}_{S h}\right) \cdot \mathbf{n}\right\|_{\mathbf{H}^{-\frac{1}{2}}\left(\partial \Omega_{S}\right)} \leq C\left\|\mathbf{u}_{S}-\mathbf{u}_{S h}\right\|_{\mathbf{H}^{\mathrm{div}}\left(\Omega_{S}\right)}
$$

From the properties of $\mathbf{V}$ and $\mathbf{V}_{h}$, we have

$$
\left(\mathbf{u}_{S}-\mathbf{u}_{S h}\right) \cdot \mathbf{n}=0, \quad \text { on } \Gamma_{S} .
$$

Therefore, following the approach of Galvis and Sarkis in [16], we have

and hence

$$
\left\|\left(\mathbf{u}_{S}-\mathbf{u}_{S h}\right) \cdot \mathbf{n}\right\|_{\mathbf{H}^{-\frac{1}{2}}\left(\Gamma_{S D}\right)} \leq C\left\|\left(\mathbf{u}_{S}-\mathbf{u}_{S h}\right) \cdot \mathbf{n}\right\|_{\mathbf{H}^{-\frac{1}{2}}\left(\partial \Omega_{S}\right)},
$$

$$
\left\|\left(\mathbf{u}_{S}-\mathbf{u}_{S h}\right) \cdot \mathbf{n}\right\|_{\mathbf{H}^{-\frac{1}{2}}\left(\Gamma_{S D}\right)} \leq C\left\|\mathbf{u}_{S}-\mathbf{u}_{S h}\right\|_{\mathbf{H}^{\mathrm{div}}\left(\Omega_{S}\right)} .
$$

On the other hand, recall the interpolation trace inequality (see for instance Brenner and Scott [9]):

$$
\forall v \in H^{1}\left(\Omega_{S}\right),\|v\|_{L^{2}\left(\partial \Omega_{S}\right)} \leq C\|v\|_{L^{2}\left(\Omega_{S}\right)}^{\frac{1}{2}}\|v\|_{H^{1}\left(\Omega_{S}\right)}^{\frac{1}{2}} .
$$

For lack of regularity, it cannot be applied to $\mathbf{u}_{S h}$. Nevertheless, in the appendix, we establish that

$$
\begin{aligned}
\forall \mathbf{v}_{h} \in \mathbf{V}_{h},\left\|\mathbf{v}_{h}\right\|_{\mathbf{L}^{2}\left(\Gamma_{S D}\right)} \leq & C\left(\left\|\mathbf{v}_{h}\right\|_{\mathbf{L}^{2}\left(\Omega_{S}\right)}+h^{\frac{1}{2}}\left(\left\|\nabla \mathbf{v}_{h}\right\|_{\mathbb{T}_{h}^{S}}^{2}+\left\|\sigma_{h} \llbracket \mathbf{v}_{h} \rrbracket\right\|_{\Gamma_{h}^{S}}^{2}\right)^{\frac{1}{2}}\right. \\
& \left.+\left\|\mathbf{v}_{h}\right\|_{\mathbf{L}^{2}\left(\Omega_{S}\right)}^{\frac{1}{2}}\left(\left\|\nabla \mathbf{v}_{h}\right\|_{\mathbb{T}_{h}^{S}}^{2}+\left\|\sigma_{h} \llbracket \mathbf{v}_{h} \rrbracket\right\|_{\Gamma_{h}^{S}}^{2}\right)^{\frac{1}{4}}\right),
\end{aligned}
$$


with a constant $C$ independent of $h$. Then we write

$$
\mathbf{u}_{S}-\mathbf{u}_{S h}=\mathbf{u}_{S}-R_{h}\left(\mathbf{u}_{S}\right)+R_{h}\left(\mathbf{u}_{S}\right)-\mathbf{u}_{S h} .
$$

We first apply (47) to $R_{h}\left(\mathbf{u}_{S}\right)-\mathbf{u}_{S h}$ :

$$
\left\|R_{h}\left(\mathbf{u}_{S}\right)-\mathbf{u}_{S h}\right\|_{L^{2}\left(\Gamma_{S D}\right)} \leq C h^{s-1+\frac{\alpha}{2}}|\mathbf{u}|_{H^{s}(\Omega)} .
$$

Given the improved accuracy in the Stokes subdomain, we can now estimate the normal trace on the interface and obtain:

Lemma 10. Let the assumptions of Theorem 9 hold. Then, the normal trace of $\mathbf{u}_{S}-\mathbf{u}_{S h}$ on $\Gamma_{S D}$ satisfies

$$
\left\|\left(\mathbf{u}_{S}-\mathbf{u}_{S h}\right) \cdot \mathbf{n}\right\|_{L^{2}\left(\Gamma_{S D}\right)} \leq C h^{s-1+\frac{\alpha}{2}} \mid \mathbf{u}_{\mathbf{H}^{s}(\Omega)} .
$$

Moreover, if $g$ in $(2 \mathrm{c})$ belongs to $H^{s-1+\alpha}(\Omega)$, we have

$$
\left\|\left(\mathbf{u}_{S}-\mathbf{u}_{S h}\right) \cdot \mathbf{n}\right\|_{H^{-\frac{1}{2}}\left(\Gamma_{S D}\right)} \leq C h^{s-1+\alpha}\left(|\mathbf{u}|_{\mathbf{H}^{s}(\Omega)}+|g|_{H^{s-1+\alpha}(\Omega)}\right) .
$$

We skip the proof that now follows easily from (40), (44), (43), and (47).

3.4. $L^{2}$ error in the Darcy subdomain. Now, that we have estimated the error in $\Omega_{S}$ and on $\Gamma_{S D}$, we are going to leave the view of a monolithic, coupled problem and consider the Darcy subproblem alone. To this end, we introduce the auxiliary function spaces $\mathbf{V}^{D}=$ $\mathbf{H}_{0}^{\text {div }}\left(\Omega_{D}\right)$ as well as the corresponding Raviart-Thomas space $\mathbf{V}_{h}^{D} \subset \mathbf{V}^{D}$. We remark that both spaces can be extended by zero to elements in $\mathbf{V}$ and $\mathbf{V}_{h}$, respectively. Additionally, we define $Q^{D}$ and $Q_{h}^{D}$ (resp. $Q^{S}$ and $Q_{h}^{S}$ ) as the restrictions of $Q$ and $Q_{h}$ to $\Omega_{D}$ (resp. $\Omega_{S}$ ). As explained in Section 2.1, we can relax the zero mean value constraint on the test functions of $Q$ and $Q_{h}$, and hence also on the test functions of $Q^{S}$ and $Q_{h}^{S}$, or $Q^{D}$ and $Q_{h}^{D}$.

As a first step towards estimating the Darcy error, we consider the following

Lemma 11. There is a function $\mathbf{w} \in \mathbf{H}^{\text {div }}\left(\Omega_{D}\right)$ satisfying $\nabla \cdot \mathbf{w}=0$ in $\Omega_{D}, \mathbf{w} \cdot \mathbf{n}=0$ on $\Gamma_{D}$ and $\mathbf{w} \cdot \mathbf{n}=\left(\mathbf{u}_{S}-\mathbf{u}_{S h}\right) \cdot \mathbf{n}$ on $\Gamma_{S D}$, such that

$$
\begin{gathered}
\|\mathbf{w}\|_{\mathbf{L}^{2}\left(\Omega_{D}\right)} \leq C\left\|\left(\mathbf{u}_{S}-\mathbf{u}_{S h}\right) \cdot \mathbf{n}\right\|_{H^{-\frac{1}{2}}\left(\Gamma_{S D}\right)}, \\
\|\mathbf{w}\|_{\mathbf{H}^{\frac{1}{2}\left(\Omega_{D}\right)}} \leq C\left\|\left(\mathbf{u}_{S}-\mathbf{u}_{S h}\right) \cdot \mathbf{n}\right\|_{L^{2}\left(\Gamma_{S D}\right)},
\end{gathered}
$$

with a constant $C$ independent of $h$.

Proof. Let $\varphi \in H^{1}\left(\Omega_{D}\right) / \mathbb{R}$ be the unique variational solution to the problem

$$
\begin{aligned}
-\Delta \varphi & =0, & & \text { in } \Omega_{D}, \\
\frac{\partial \varphi}{\partial n} & =0, & & \text { on } \Gamma_{D}, \\
\frac{\partial \varphi}{\partial n} & =\left(\mathbf{u}_{S}-\mathbf{u}_{S h}\right) \cdot \mathbf{n}, & & \text { on } \Gamma_{S D},
\end{aligned}
$$

where exceptionally the normal derivative of $\varphi$ is defined with the normal vector pointing inside $\Omega_{D}$, so as to coincide with $\mathbf{n}$ on $\Gamma_{S D}$. This problem has a unique solution $\varphi \in$ $H^{1}\left(\Omega_{D}\right) / \mathbb{R}$ because, as

$$
\forall q_{h} \in Q_{h}^{S},\left(\nabla \cdot\left(\mathbf{u}_{S}-\mathbf{u}_{S h}\right), q_{h}\right)_{\Omega_{S}}=0,
$$

we have in particular,

$$
\left(\nabla \cdot\left(\mathbf{u}_{S}-\mathbf{u}_{S h}\right), 1\right)_{\Omega_{S}}=0 .
$$


Then the boundary conditions on $\mathbf{V}$ and $\mathbf{V}_{h}$ imply that

$$
\int_{\Gamma_{S D}}\left(\mathbf{u}_{S}-\mathbf{u}_{S h}\right) \cdot \mathbf{n}=0
$$

Thus

$$
|\varphi|_{H^{1}\left(\Omega_{D}\right)} \leq C\|\nabla \varphi \cdot \mathbf{n}\|_{H^{-\frac{1}{2}}\left(\partial \Omega_{D}\right)} \leq C\left\|\left(\mathbf{u}_{S}-\mathbf{u}_{S h}\right) \cdot \mathbf{n}\right\|_{H^{-\frac{1}{2}}\left(\Gamma_{S D}\right)}
$$

Moreover, with boundary data in $L^{2}\left(\partial \Omega_{D}\right)$, we have $\varphi \in H^{\frac{3}{2}}\left(\Omega_{D}\right)$ and we have the following bound [26]

$$
|\varphi|_{H^{\frac{3}{2}}\left(\Omega_{D}\right)} \leq C\left\|\left(\mathbf{u}_{S}-\mathbf{u}_{S h}\right) \cdot \mathbf{n}\right\|_{L^{2}\left(\Gamma_{S D}\right)} .
$$

To conclude, it suffices to take $\mathbf{w}=\nabla \varphi$.

With this lemma and the estimate for the trace in the previous subsection, we are ready to prove the main result for the Darcy subregion.

Theorem 12. Let Assumptions $1-4$ hold with the constant $\left.\alpha \in] \frac{1}{2}, 1\right]$ determined by Assumption 1. Let $\mathbf{u} \in \mathbf{H}^{s}(\Omega)$ for $\left.\left.s \in\right] \frac{3}{2}, k+1\right]$ be the solution of (7) and let $\mathbf{u}_{h}$ be the solution to (39). Moreover, let $g \in H^{s-1+\alpha}(\Omega)$ in (2c). Then we have the estimate for the error in the Darcy subdomain:

$$
\left\|\mathbf{u}_{D}-\mathbf{u}_{D h}\right\|_{L^{2}\left(\Omega_{D}\right)} \leq C h^{s}\left(|\mathbf{u}|_{H^{s-1+\alpha}(\Omega)}+|g|_{H^{s-1+\alpha}(\Omega)}\right) .
$$

Proof. We observe that $\mathbf{u}_{D}$ solves the problem

$$
\begin{aligned}
a_{D}\left(\mathbf{u}_{D}, \mathbf{v}\right)+b\left(\mathbf{v}, p_{D}\right) & =(\mathbf{f}, \mathbf{v})_{\Omega_{D},} & & \forall \mathbf{v} \in \mathbf{V}^{D}, \\
b\left(\mathbf{u}_{D}, q\right) & =-(g, q)_{\Omega_{D}}, & & \forall q \in Q^{D}, \\
\mathbf{u}_{D} \cdot \mathbf{n} & =0, & & \text { on } \Gamma_{D}, \\
\mathbf{u}_{D} \cdot \mathbf{n} & =\mathbf{u}_{S} \cdot \mathbf{n}, & & \text { on } \Gamma_{S D} .
\end{aligned}
$$

Similarly, $\mathbf{u}_{D h}$ solves

$$
\begin{aligned}
a_{D}\left(\mathbf{u}_{D h}, \mathbf{v}\right)+b\left(\mathbf{v}, p_{D h}\right) & =(\mathbf{f}, \mathbf{v})_{\Omega_{D}}, & & \forall \mathbf{v} \in \mathbf{V}_{h}^{D}, \\
b\left(\mathbf{u}_{D h}, q\right) & =-(g, q)_{\Omega_{D}}, & & \forall q \in Q_{h}^{D}, \\
\mathbf{u}_{D h} \cdot \mathbf{n} & =0, & & \text { on } \Gamma_{D}, \\
\mathbf{u}_{D h} \cdot \mathbf{n} & =\mathbf{u}_{S h} \cdot \mathbf{n}, & & \text { on } \Gamma_{S D} .
\end{aligned}
$$

Substracting (56) to (60) and adding and substracting the interpolant $R_{h}\left(\mathbf{u}_{D}-\mathbf{w}\right)$, where $\mathbf{w}$ is defined in Lemma 11, yields the error equation for all $\mathbf{v}_{h} \in \mathbf{V}_{h}^{D}$ :

$$
\begin{aligned}
a_{D}\left(\mathbf{u}_{D h}-R_{h}\left(\mathbf{u}_{D}\right)+R_{h}(\mathbf{w}), \mathbf{v}_{h}\right)+ & a_{D}\left(R_{h}\left(\mathbf{u}_{D}\right)-\mathbf{u}_{D}, \mathbf{v}_{h}\right) \\
& -a_{D}\left(R_{h}(\mathbf{w}), \mathbf{v}_{h}\right)+b\left(\mathbf{v}_{h}, p_{D h}-p_{D}\right)=0 .
\end{aligned}
$$

We choose $\mathbf{v}_{h}=\mathbf{u}_{D h}-R_{h}\left(\mathbf{u}_{D}\right)+R_{h}(\mathbf{w})$ and we note that $\mathbf{v}_{h} \in \mathbf{V}_{h}^{D}$. Indeed, we have

$$
\left(\mathbf{u}_{D h}-\mathbf{u}_{D}+\mathbf{w}\right) \cdot \mathbf{n}=0, \quad \text { on } \partial \Omega_{D},
$$

and with (26), this implies

$$
\mathbf{v}_{h} \cdot \mathbf{n}=R_{h}\left(\mathbf{u}_{D h}-\mathbf{u}_{D}+\mathbf{w}\right) \cdot \mathbf{n}=0 .
$$

From (21), we have

$$
\nabla \cdot \mathbf{v}_{h}=\nabla \cdot\left(\mathbf{u}_{D h}-R_{h}\left(\mathbf{u}_{D}\right)\right)+\nabla \cdot R_{h}(\mathbf{w})=0 .
$$


This implies $b\left(\mathbf{v}_{h}, p_{D h}-p_{D}\right)=0$. We then have, with a constant $C$ that depends on the permeability tensor of the porous media only:

$$
\left\|\mathbf{u}_{D h}-R_{h}\left(\mathbf{u}_{D}\right)+R_{h}(\mathbf{w})\right\|_{L^{2}\left(\Omega_{D}\right)} \leq C\left(\left\|R_{h}\left(\mathbf{u}_{D}\right)-\mathbf{u}_{D}\right\|_{L^{2}\left(\Omega_{D}\right)}+\left\|R_{h}(\mathbf{w})\right\|_{L^{2}\left(\Omega_{D}\right)}\right) .
$$

Using the triangle inequality and property (22), we have

$$
\begin{aligned}
& \left\|R_{h}(\mathbf{w})\right\|_{L^{2}\left(\Omega_{D}\right)} \leq\left\|R_{h}(\mathbf{w})-\mathbf{w}\right\|_{L^{2}\left(\Omega_{D}\right)}+\|\mathbf{w}\|_{L^{2}\left(\Omega_{D}\right)} \\
& \leq C h^{\frac{1}{2}}\|\mathbf{w}\|_{H^{\frac{1}{2}\left(\Omega_{D}\right)}}+\|\mathbf{w}\|_{L^{2}\left(\Omega_{D}\right)} .
\end{aligned}
$$

From (50) and (51), we obtain

$$
\left\|R_{h}(\mathbf{w})\right\|_{L^{2}\left(\Omega_{D}\right)} \leq C h^{\frac{1}{2}}\left\|\left(\mathbf{u}_{S}-\mathbf{u}_{S h}\right) \cdot \mathbf{n}\right\|_{L^{2}\left(\Gamma_{S D}\right)}+C\left\|\left(\mathbf{u}_{S}-\mathbf{u}_{S h}\right) \cdot \mathbf{n}\right\|_{H^{-\frac{1}{2}}\left(\Gamma_{S D}\right)} .
$$

We can then conclude with Lemma 10 and with property (22) of the Raviart-Thomas interpolant.

\section{APPENDIX}

This section is devoted to the proof of the discrete interpolation trace inequality (47) with a constant $C$ independent of $h$. More generally, the result is valid for any space of piecewise polynomial functions with degree in a fixed range, say

$$
X_{h}=\left\{v_{h} \in L^{2}\left(\Omega_{S}\right)\left|\forall T \in \mathbb{T}_{h}^{S}, v_{h}\right|_{T} \in \mathbb{P}_{k}\right\},
$$

where $k$ may vary from one element to the next, but stays within a fixed range, such as $k_{m} \leq k \leq k_{M}$. To simplify, we introduce the broken seminorm on $X_{h}$ :

$$
\left|v_{h}\right|_{1, h}=\left(\left\|\nabla v_{h}\right\|_{\mathbb{T}_{h}^{S}}^{2}+\left\|\sigma_{h} \llbracket v_{h} \rrbracket\right\|_{\Gamma_{h}^{S}}^{2}\right)^{\frac{1}{2}},
$$

and we shall prove that for all $v_{h} \in X_{h}$

$$
\left\|v_{h}\right\|_{L^{2}\left(\Gamma_{S D}\right)} \leq C\left(\left\|v_{h}\right\|_{L^{2}\left(\Omega_{S}\right)}+h^{\frac{1}{2}}\left|v_{h}\right|_{1, h}+\left\|v_{h}\right\|_{L^{2}\left(\Omega_{S}\right)}^{\frac{1}{2}}\left|v_{h}\right|_{1, h}^{\frac{1}{2}}\right) .
$$

Our argument is based on an $H^{1}\left(\Omega_{S}\right)$ regularization of $v_{h}$, following ideas of $[6,21,22]$.

Let $\mathcal{P}_{h}$ denote the set of vertices of $\mathbb{T}_{h}^{S} \cap \bar{\Omega}_{S}$. We associate with each vertex a of $\mathcal{P}_{h}$ an element $T_{\mathbf{a}}$ of $\mathbb{T}_{h}^{S}$ that has vertex a (repetitions are allowed), and set

$$
\begin{gathered}
E_{h}\left(v_{h}\right)(\mathbf{a})=\left.v_{h}\right|_{T_{\mathbf{a}}}(\mathbf{a}), \\
\forall \mathbf{x} \in \bar{\Omega}_{S}, E_{h}\left(v_{h}\right)(\mathbf{x})=\sum_{\mathbf{a} \in \mathcal{P}_{h}} E_{h}\left(v_{h}\right)(\mathbf{a}) \varphi_{\mathbf{a}}(\mathbf{x}),
\end{gathered}
$$

where $\varphi_{\mathbf{a}}$ is the standard, globally continuous basis function, that is $\mathbb{P}_{1}$ in each $T$, and takes the value 1 at $\mathbf{a}$ and zero at all other vertices of $\mathcal{P}_{h}$. As $E_{h}\left(v_{h}\right)$ belongs to $H^{1}\left(\Omega_{S}\right)$, it satisfies (46) with $\Gamma_{S D}$ instead of $\partial \Omega_{S}$ :

$$
\left\|E_{h}\left(v_{h}\right)\right\|_{L^{2}\left(\Gamma_{S D}\right)} \leq C\left\|E_{h}\left(v_{h}\right)\right\|_{L^{2}\left(\Omega_{S}\right)}^{\frac{1}{2}}\left\|E_{h}\left(v_{h}\right)\right\|_{H^{1}\left(\Omega_{S}\right)}^{\frac{1}{2}},
$$

with a constant $C$ that only depends on $\Omega_{S}$ and $\Gamma_{S D}$. By inserting $E_{h}\left(v_{h}\right)$ and expanding the $H^{1}$ norm, this becomes

$$
\begin{aligned}
\left\|v_{h}\right\|_{L^{2}\left(\Gamma_{S D}\right)} \leq & \left\|v_{h}-E_{h}\left(v_{h}\right)\right\|_{L^{2}\left(\Gamma_{S D}\right)} \\
& +C\left(\left\|E_{h}\left(v_{h}\right)\right\|_{L^{2}\left(\Omega_{S}\right)}+\left\|E_{h}\left(v_{h}\right)\right\|_{L^{2}\left(\Omega_{S}\right)}^{\frac{1}{2}}\left\|\nabla E_{h}\left(v_{h}\right)\right\|_{\mathbf{L}^{2}\left(\Omega_{S}\right)}^{\frac{1}{2}}\right) .
\end{aligned}
$$


We must evaluate the terms in the right-hand side of (66). To estimate $\nabla E_{h}\left(v_{h}\right)$, we use the following auxiliary lemma; to be specific, we treat the case $d=3$. The twodimensional case is similar.

Lemma 13. For $\ell=1$ or $\ell=-1$, let $\hat{T}_{\ell}$ denote the two adjacent reference elements with vertices $\hat{\mathbf{a}}_{0}=(0,0,0), \hat{\mathbf{a}}_{2}=(0,1,0), \hat{\mathbf{a}}_{3}=(0,0,1)$, and $\hat{\mathbf{a}}_{\ell}=(\ell, 0,0)$, let $\hat{F}$ denote their common face and let $\hat{v}$ be such that it is a polynomial of degree $k_{\ell}$ in $\hat{T}_{\ell}$. There exists a constant $\hat{C}$, only depending on $k_{\ell}$ and the reference face $\hat{F}$ such that

$$
|\hat{v}|_{\hat{T}_{1}}\left(\hat{\mathbf{a}}_{0}\right)-\left.\hat{v}\right|_{\hat{T}_{-1}}\left(\hat{\mathbf{a}}_{0}\right) \mid \leq \hat{C}\left\|[\hat{v}]_{\hat{F}}\right\|_{L^{2}(\hat{F})} .
$$

Proof. Let $\hat{w}=\left.\left(\left.\hat{v}\right|_{\hat{T}_{1}}-\left.\hat{v}\right|_{\hat{T}_{-1}}\right)\right|_{\hat{F}}$. Then

$$
\left|\hat{w}\left(\hat{\mathbf{a}}_{0}\right)\right| \leq\|\hat{w}\|_{L^{\infty}(\hat{F})}=\left\|[\hat{v}]_{\hat{F}}\right\|_{L^{\infty}(\hat{F})},
$$

and (67) follows from the fact that $[\hat{v}]_{\hat{F}}$ is a polynomial of degree $\max \left(k_{\ell}\right)$ and all norms are equivalent in finite dimensional spaces.

Lemma 14. There exists a constant $C$, independent of $h$, such that

$$
\forall v_{h} \in X_{h},\left\|\nabla E_{h}\left(v_{h}\right)\right\|_{L^{2}\left(\Omega_{S}\right)} \leq C\left|v_{h}\right|_{1, h} .
$$

Proof. Let $T$ be an element of $\mathbb{T}_{h}^{S}$ with vertices $\mathbf{a}_{i}, 1 \leq i \leq d+1$. We have

$$
\left\|\nabla E_{h}\left(v_{h}\right)\right\|_{L^{2}(T)}^{2}=\left\|\sum_{i=1}^{d+1} E_{h}\left(v_{h}\right)\left(\mathbf{a}_{i}\right) \nabla \varphi_{\mathbf{a}_{i}}\right\|_{L^{2}(T)}^{2} .
$$

But

$$
\forall \mathbf{x} \in T, \sum_{i=1}^{d+1} \varphi_{\mathbf{a}_{i}}(\mathbf{x})=1
$$

hence

$$
\forall \mathbf{x} \in T, \sum_{i=1}^{d+1} \nabla \varphi_{\mathbf{a}_{i}}(\mathbf{x})=\mathbf{0}
$$

and we can write

$$
\begin{aligned}
\left\|\nabla E_{h}\left(v_{h}\right)\right\|_{L^{2}(T)}^{2} & =\left\|\sum_{i=1}^{d+1}\left(E_{h}\left(v_{h}\right)\left(\mathbf{a}_{i}\right)-v_{h}\left(\mathbf{a}_{1}\right)\right) \nabla \varphi \mathbf{a}_{i}\right\|_{L^{2}(T)}^{2} \\
& \leq\left(\sum_{i=1}^{d+1}\left|v_{h}\right|_{T_{\mathbf{a}_{i}}}\left(\mathbf{a}_{i}\right)-\left.v_{h}\right|_{T}\left(\mathbf{a}_{1}\right) \mid\left\|\nabla \varphi_{\mathbf{a}_{i}}\right\|_{L^{2}(T)}\right)^{2} .
\end{aligned}
$$

Given a vertex $\mathbf{a}_{i}$, there exists a sequence of two-by-two adjacent elements $T_{1}, T_{2}, \ldots, T_{j}$ that share this vertex, and such that $T_{1}=T_{\mathbf{a}_{i}}, T_{j}=T$. Therefore

$$
\left.v_{h}\right|_{T_{\mathbf{a}_{i}}}\left(\mathbf{a}_{i}\right)-\left.v_{h}\right|_{T}\left(\mathbf{a}_{1}\right)=\sum_{n=1}^{j-1}\left(\left.v_{h}\right|_{T_{n}}-\left.v_{h}\right|_{T_{n+1}}\right)\left(\mathbf{a}_{i}\right)+\left.v_{h}\right|_{T}\left(\mathbf{a}_{i}\right)-\left.v_{h}\right|_{T}\left(\mathbf{a}_{1}\right) .
$$

On one hand, Lemma 13 implies that

$$
\left|\left(\left.v_{h}\right|_{T_{n}}-\left.v_{h}\right|_{T_{n+1}}\right)\left(\mathbf{a}_{i}\right)\right| \leq \hat{C}\left(\frac{|\hat{F}|}{\left|F_{n}\right|}\right)^{\frac{1}{2}}\left\|\left[v_{h}\right]_{F_{n}}\right\|_{L^{2}\left(F_{n}\right)},
$$

where $F_{n}$ is the face shared by $T_{n}$ and $T_{n+1}$. On the other hand, if $\mathbf{a}_{i} \neq \mathbf{a}_{1}$, by passing to the reference element $\hat{T}$, and using equivalence of norms, we have

$$
\left|v_{h}\left(\mathbf{a}_{i}\right)-v_{h}\left(\mathbf{a}_{1}\right)\right| \leq h_{\hat{T}}\|\hat{\nabla} \hat{v}\|_{L^{\infty}(\hat{T})} \leq \hat{C}\|\hat{\nabla} \hat{v}\|_{L^{2}(\hat{T})} .
$$


Thus

$$
\left|v_{h}\left(\mathbf{a}_{i}\right)-v_{h}\left(\mathbf{a}_{1}\right)\right| \leq \hat{C} h_{T}\left(\frac{|\hat{T}|}{|T|}\right)^{\frac{1}{2}}\left\|\nabla v_{h}\right\|_{L^{2}(T)}
$$

By substituting these two inequalities into (70) and evaluating the basis functions in the reference element, we obtain

$\left\|\nabla E_{h}\left(v_{h}\right)\right\|_{L^{2}(T)}^{2} \leq \hat{C}\left[\sum_{i=1}^{d+1}\left(\sum_{n=1}^{j-1} \frac{1}{\left|F_{n}\right|}\left\|\left[v_{h}\right]_{F_{n}}\right\|_{L^{2}\left(F_{n}\right)}^{2}+\frac{h_{T}^{2}}{|T|}\left\|\nabla v_{h}\right\|_{L^{2}(T)}^{2}\right) \frac{|T|}{\rho_{T}^{2}}\left\|\hat{\nabla} \hat{\varphi}_{i}\right\|_{L^{2}(\hat{T})}^{2}\right]$,

where $\hat{C}$ takes into account the maximum number of edges or faces that meet at the vertices of $\mathcal{P}_{h}$; this number is bounded by a constant independent of $h$ since the family of triangulations is regular. We recover (68) by multiplying and dividing the first term of this sum with $\left(\sigma_{h}^{F}\right)^{2}$ and using again the regularity of the family $\mathbb{T}_{h}^{S}$.

To estimate $E_{h}\left(v_{h}\right)$, it is convenient to break it into $E_{h}\left(v_{h}\right)-v_{h}$ and $v_{h}$.

Lemma 15. There exists a constant $C$, independent of $h$, such that

$$
\forall v_{h} \in X_{h},\left\|E_{h}\left(v_{h}\right)-v_{h}\right\|_{L^{2}\left(\Omega_{S}\right)} \leq C h\left|v_{h}\right|_{1, h} .
$$

Proof. We briefly sketch the proof. Let $T$ be an element of $\mathbb{T}_{h}^{S}$ with vertices $\mathbf{a}_{i}, 1 \leq i \leq$ $d+1$. We have

$$
\left\|E_{h}\left(v_{h}\right)-v_{h}\right\|_{L^{2}(T)}^{2}=\int_{T}\left(\sum_{i=1}^{d+1}\left(E_{h}\left(v_{h}\right)\left(\mathbf{a}_{i}\right)-v_{h}(\mathbf{x})\right) \varphi_{\mathbf{a}_{i}}(\mathbf{x})\right)^{2} d \mathbf{x}
$$

an expression similar to (69) with $\mathbf{x}$ instead of $\mathbf{a}_{1}$ and the basis function instead of its gradient. As $\mathbf{x}$ and $\mathbf{a}_{1}$ are both points in $T$, the argument of Lemma 14 yields

$\left\|E_{h}\left(v_{h}\right)-v_{h}\right\|_{L^{2}(T)}^{2} \leq \hat{C}\left[\sum_{i=1}^{d+1}\left(\sum_{n=1}^{j-1} \frac{1}{\left|F_{n}\right|}\left\|\left[v_{h}\right]_{F_{n}}\right\|_{L^{2}\left(F_{n}\right)}^{2}+\frac{h_{T}^{2}}{|T|}\left\|\nabla v_{h}\right\|_{L^{2}(T)}^{2}\right)|T|\left\|\hat{\varphi}_{i}\right\|_{L^{2}(\hat{T})}^{2}\right]$,

whence (71).

It remains to evaluate $v_{h}-E_{h}\left(v_{h}\right)$ on $\Gamma_{S D}$.

Lemma 16. There exists a constant $C$, independent of $h$, such that

$$
\forall v_{h} \in X_{h},\left\|E_{h}\left(v_{h}\right)-v_{h}\right\|_{L^{2}\left(\Gamma_{S D}\right)} \leq C h^{\frac{1}{2}}\left|v_{h}\right|_{1, h}
$$

Proof. Again, we briefly sketch the proof. Let $F$ be a face of $\mathbb{T}_{h}^{S} \cap \Gamma_{S D}$ with vertices $\mathbf{a}_{i}$, $1 \leq i \leq d$. Since

$$
\forall \mathbf{x} \in F, \sum_{i=1}^{d} \varphi_{\mathbf{a}_{i}}(\mathbf{x})=1
$$

we can write

$$
\left\|E_{h}\left(v_{h}\right)-v_{h}\right\|_{L^{2}(F)}^{2}=\int_{F}\left(\sum_{i=1}^{d}\left(E_{h}\left(v_{h}\right)\left(\mathbf{a}_{i}\right)-v_{h}(\mathbf{x})\right) \varphi_{\mathbf{a}_{i}}(\mathbf{x})\right)^{2} d s(\mathbf{x}) .
$$

Therefore, the argument of Lemma 14 yields

$$
\left\|E_{h}\left(v_{h}\right)-v_{h}\right\|_{L^{2}(F)}^{2} \leq \hat{C}\left[\sum_{i=1}^{d}\left(\sum_{n=1}^{j-1} \frac{1}{\left|F_{n}\right|}\left\|\left[v_{h}\right]_{F_{n}}\right\|_{L^{2}\left(F_{n}\right)}^{2}+\frac{h_{T}^{2}}{|T|}\left\|\nabla v_{h}\right\|_{L^{2}(T)}^{2}\right)|F|\left\|\hat{\varphi}_{i}\right\|_{L^{2}(\hat{F})}^{2}\right],
$$

and (72) follows easily from this. 
By substituting (68), (71), and (72) into (66), we immediately derive the discrete interpolation trace inequality.

Theorem 17. If the family of triangulations $\mathbb{T}_{h}^{S}$ is regular, there exists a constant $C$, independent of $h$, such that

$$
\forall v_{h} \in X_{h},\left\|v_{h}\right\|_{L^{2}\left(\Gamma_{S D}\right)} \leq C\left(\left\|v_{h}\right\|_{L^{2}\left(\Omega_{S}\right)}+h^{\frac{1}{2}}\left|v_{h}\right|_{1, h}+\left\|v_{h}\right\|_{L^{2}\left(\Omega_{S}\right)}^{\frac{1}{2}}\left|v_{h}\right|_{1, h}^{\frac{1}{2}}\right) .
$$

\section{REFERENCES}

[1] R. A. Adams and J. J. F. Fournier. Sobolev Spaces, volume 140 of Pure and Applied Mathematics. Academic Press, second edition edition, 2003.

[2] T. Arbogast and D. Brunson. A computational method for approximating a Darcy-Stokes system governing a vuggy porous medium. Computational Geosciences, 11:207-218, 2007.

[3] D. N. Arnold, D. Boffi, and R. S. Falk. Approximation by quadrilateral finite elements. Math. Comput., 71:909-922, 2002.

[4] D. N. Arnold, F. Brezzi, B. Cockburn, and D. Marini. Unified analysis of discontinuous Galerkin methods for elliptic problems. SIAM J. Numer. Anal., 39(5):1749-1779, 2002.

[5] G. S. Beavers and D. D. Joseph. Boundary conditions at a naturally impermeable wall. J. Fluid. Mech, 30:197-207, 1967.

[6] S. C. Brenner. Poincaré-Friedrichs inequalities for piecewise $H^{1}$ functions. SIAM J. Numer. Anal., 41(1):306-324, 2003.

[7] S. C. Brenner. Discrete Sobolev and Poincaré inequalities for piecewise polynomial functions. Electron. Trans. Numer. Anal., 18:42-48, 2004.

[8] S. C. Brenner. Korn's inequalities for piecewise $h^{1}$ vector fields. Math. Comput., 73(247):1067-1087, 2004.

[9] S. C. Brenner and L. R. Scott. The Mathematical Theory of Finite Element Methods. Springer, 2nd edition, 2002.

[10] F. Brezzi and M. Fortin. Mixed and Hybrid Finite Element Methods. Springer, 1991.

[11] P. G. Ciarlet. Basic error estimates for elliptic problems - finite element methods, part 1. In P. G. Ciarlet and J.-L. Lions, editors, Handbook of Numerical Analysis, volume II, pages 17-352. Elsevier/North-Holland, Amsterdam, 1991.

[12] B. Cockburn, G. Kanschat, and D. Schötzau. A locally conservative LDG method for the incompressible Navier-Stokes equations. Math. Comput., 74:1067-1095, 2005.

[13] B. Cockburn, G. Kanschat, and D. Schötzau. A note on discontinuous Galerkin divergence-free solutions of the Navier-Stokes equations. J. Sci. Comput., 31(1-2):61-73, 2007.

[14] B. Cockburn, G. Kanschat, D. Schötzau, and C. Schwab. Local discontinuous Galerkin methods for the Stokes system. SIAM J. Numer. Anal., 40(1):319-343, 2002.

[15] M. Discacciati and A. Quarteroni. Navier-Stokes/Darcy coupling: modeling, analysis, and numerical approximation. Rev. Mat. Complut., 22(2):315-426, 2009.

[16] J. Galvis and M. Sarkis. Non-matching mortar discretization analysis for the coupling Stokes-Darcy equations. Electron. Trans. Numer. Anal., 26:350-384, 2007.

[17] G. N. Gatica, R. Oyarzúa, and F.-J. Sayas. Analysis of fully-mixed finite element methods for the StokesDarcy coupled problem. Math. Comput., 80(276):1911-1948, 2011.

[18] G. N. Gatica, R. Oyarzúa, and F.-J. Sayas. Convergence of a family of Galerkin discretizations for the Stokes-Darcy coupled problem. Numer. Methods Partial Differential Equations, 27(3):721-748, 2011.

[19] V. Girault and P.-A. Raviart. Finite element approximations of the Navier-Stokes equations. Springer, 1986.

[20] V. Girault and B. Rivière. DG approximation of coupled Navier-Stokes and Darcy equations by BeaverJoseph-Saffman interface condition. SIAM J. Numer. Anal., 47:2052-2089, 2009.

[21] V. Girault, B. Rivière, and M. F. Wheeler. A splitting method using discontinuous Galerkin for the transient incompressible Navier-Stokes equations. M2AN Math. Model. Numer. Anal., 39(6):1115-1147, 2005.

[22] V. Girault and M. Wheeler. Numerical discretization of a Darcy-Forchheimer model. Numerische Mathematik, 110:161-198, 2008.

[23] P. Hansbo and M. G. Larson. Discontinuous Galerkin methods for incompressible and nearly incompressible elasticity by Nitsche's method. Comput. Methods Appl. Mech. Eng., 191:1895-1908, 2002.

[24] N. Hanspal, A. Waghode, V. Nassehi, and R. Wakeman. Numerical analysis of coupled Stokes/Darcy flows in industrial filtrations. Transport in Porous Media, 64(1):1573-1634, 2006.

[25] W. Jäger and A. Mikelić. Modeling effective interface laws for transport phenomena between an unconfined fluid and a porous medium using homogenization. Transp. Porous Media, 78(3):489-508, 2009. 
[26] D. S. Jerrison and C. Kenig. The Neumann problem on Lipschitz domains. Bull. AMS, 4:203-207, 1981.

[27] G. Kanschat. Discontinuous Galerkin Methods for Viscous Flow. Deutscher Universitätsverlag, Wiesbaden, 2007.

[28] G. Kanschat and B. Rivière. A strongly conservative finite element method for the coupling of Stokes and Darcy flow. J. Comput. Phys., 229:5933-5943, 2010.

[29] W. J. Layton, F. Schieweck, and I. Yotov. Coupling fluid flow with porous media flow. SIAM J. Numer. Anal., 40(6):2195-2218, 2003.

[30] J.-L. Lions and E. Magenes. Nonhomogeneous Boundary Value Problems and Applications. Springer, 1972.

[31] J. Nečas. Les méthodes directes en théorie des équations elliptiques. Masson, Paris, 1967.

[32] P.-A. Raviart and J. M. Thomas. A mixed method for second order elliptic problems. In I. Galligani and E. Magenes, editors, Mathematical Aspects of the Finite Element Method, pages 292-315. Springer, New York, 1977.

[33] B. Rivière. Discontinuous Galerkin Methods for Solving Elliptic and Parabolic Equations. Frontiers in applied Mathematics. SIAM, 2008.

[34] B. Rivière, M. F. Wheeler, and V. Girault. A priori error estimates for finite element methods based on discontinuous approximation spaces for elliptic problems. SIAM J. Numer. Anal., 39(3):902-931, 2001.

[35] B. Rivière and I. Yotov. Locally conservative coupling of Stokes and Darcy flow. SIAM J. Numer. Anal., 42:1959-1977, 2005.

[36] P. Saffman. On the boundary condition at the surface of a porous media. Stud. Appl. Math., 50:292-315, 1971.

[37] D. Schötzau, C. Schwab, and A. Toselli. hp-DGFEM for incompressible flows. SIAM J. Numer. Anal., 40:2171-2194, 2003.

[38] J. Wang and X. Ye. New finite element methods in computational fluid dynamics by $H$ (div) elements. SIAM J. Numer. Anal., 45:1269-1286, 2007.

(V. Girault) Department of Mathematics, Texas A\&M University, 3368 TAMU, College StaTION, TX 77843

Current address: UPMC-Paris 6 and CNRS, UMR 7598, F-75230 Paris Cedex 05, France

E-mail address: giraulteann.jussieu.fr

(G. Kanschat) Department of Mathematics, Texas A\&M University, 3368 TAMU, College STATION, TX 77843

E-mail address: dr.guido.kanschat@gmail.com

(B. Rivière) Department of Computational \& Applied Mathematics, Rice University, 6100 MAIN STREeT, MS-134, Houston, TX 77005-1892

E-mail address: riviere@caam.rice.edu 